\title{
REPRESENTATIONS OF THE ALTERNATING GROUP WHICH ARE IRREDUCIBLE OVER SUBGROUPS. II
}

\author{
ALEXANDER KLESHCHEV, PETER SIN, AND PHAM HUU TIEP
}

\begin{abstract}
We prove that non-trivial representations of the alternating group $A_{n}$ are reducible over a primitive proper subgroup which is isomorphic to some alternating group $A_{m}$. A similar result is established for finite simple classical groups embedded in $A_{n}$ via their standard rank 3 permutation representations.
\end{abstract}

\section{INTRODUCTION}

If $\Gamma$ is a transitive permutation group with a point stabilizer $X$ then $\Gamma$ is primitive if and only if $X<\Gamma$ is a maximal subgroup. So studying primitive permutation groups is equivalent to studying maximal subgroups. In most problems involving a finite primitive group $\Gamma$, the Aschbacher-O'Nan-Scott theorem [AS] allows one to concentrate on the case where $\Gamma$ is almost quasi-simple, i.e. $L \triangleleft \Gamma / Z(\Gamma) \leq \operatorname{Aut}(L)$ for a non-abelian simple group L. The results of Liebeck-Praeger-Saxl [LPS] and Liebeck-Seitz [LS] then allow one to assume furthermore that $\Gamma$ is a finite classical group.

In the latter case, the possible structure of maximal subgroups $X$ is described by Aschbacher's theorem [A]: if $X<\Gamma$ is maximal then $X$ belongs to

$$
\mathcal{S} \cup \bigcup_{i=1}^{8} \mathcal{C}_{i},
$$

where $\mathcal{C}_{1}, \ldots, \mathcal{C}_{8}$ are collections of certain explicit natural subgroups of $\Gamma$, and $\mathcal{S}$ is the collection of almost quasi-simple groups that act absolutely irreducibly on the natural module for the classical group $\Gamma$.

It is not true, however, that every subgroup $X$ in (1.1) is actually maximal in $\Gamma$. For $X \in \cup_{i=1}^{8} \mathcal{C}_{i}$, the maximality of $X$ has been determined by Kleidman-Liebeck [KlL] (see also [BDR] for a complete classification of maximal subgroups in low-dimensional finite classical groups). So let $X \in \mathcal{S}$. If $X$ is not maximal then $X<G<\Gamma$ for a certain maximal subgroup $G$ in $\Gamma$. The most challenging case to handle is when $G \in \mathcal{S}$ as well. This motivates the following problem, where $\mathbb{F}$ is an algebraically closed field of characteristic $p \geq 0$ :

2000 Mathematics Subject Classification: 20C20, 20C30, 20B35, $20 \mathrm{~B} 15$.

The first author was supported by the NSF (grant DMS-1161094) and the Humboldt Foundation. The second author was partially supported by the Simons Foundation (grant \#204181). The third author was supported by the NSF (grants DMS-0901241 and DMS-1201374) and the Simons Foundation Fellowship 305247.

The authors are grateful to the referees for careful reading and helpful comments on the paper. 
Problem 1.1. Classify all triples $(G, V, X)$ where $G$ is an almost quasi-simple finite group, $V$ is an $\mathbb{F} G$-module of dimension greater than one, and $X$ is a proper subgroup of $G$ such that the restriction $\operatorname{Res}_{X}^{G} V$ is irreducible.

Many results have been obtained concerning various cases of Problem 1.1 - see for instance [KT2] and references therein. In this paper, we are mostly interested in the case $G$ is the alternating group $G=\mathrm{A}_{n}$ or the symmetric group $\mathrm{S}_{n}$. In this case, under the assumption $p>3$ (or $p=0$ ), Problem 1.1 has been solved in [BK, KS2] - see also [KT1] for double-covers $\hat{\mathrm{A}}_{n}$ and $\hat{\mathrm{S}}_{n}$ and [S, KlW] for the characteristic zero case. A number of techniques employed in these papers unfortunately break down in the case $(G, X)=\left(\mathrm{A}_{n}, \mathrm{~A}_{m}\right)$ and $p=2,3$ (and especially when $X$ is a primitive subgroup of $G$ ). On the other hand, this case is of crucial importance in a number of applications. The purpose of this paper is to resolve this important case, and our main result is:

Theorem 1.2. Let $X \cong \mathrm{A}_{m}$ be a primitive subgroup of $\mathrm{A}_{n}$ with $n>m \geq 9$. Let $\mathbb{F}$ be an algebraically closed field of arbitrary characteristic and $V$ be a non-trivial $\mathbb{F} \mathrm{A}_{n}$-module. Then $V$ is reducible over $X$.

The bound $m \geq 9$ is the best possible - see Remark 8.2 and Lemma 8.1.

We emphasize that our methods also apply to many other primitive subgroups of $A_{n}$. To illustrate this, in this paper we handle the simple classical groups $X$ that embed in $A_{n}$ via their standard rank 3 permutation representations:

Theorem 1.3. Let $X=L / Z(L)$ be a finite simple classical group, where $L$ is one of the following group: $S L_{d}(q), S U_{d}(q)$, or $S p_{d}(q)^{\prime}$ with $d \geq 4$, and $\Omega_{d}^{ \pm}(q)$ with $d \geq 5$. Let $W$ denote the natural d-dimensional module for $L$, and let $X$ be embedded in $\operatorname{Sym}(\Omega)=\mathrm{S}_{n}$ via its rank 3 permutation action on the set $\Omega$ of 2-dimensional subspaces of $W$ in the case $L=S L_{d}(q)$, and of 1-dimensional singular subspaces of $W$ otherwise. If $V$ is any $\mathbb{F} \mathrm{A}_{n}$-module of dimension $>1$, then $\operatorname{Res}_{X}^{\mathrm{A}_{n}} V$ is reducible.

We plan to extend this result to the remaining simple primitive subgroups of $A_{n}$ in a sequel. Together with the results of [BK, KS2] and the current paper, this will completely solve Problem 1.1 for $G=\mathrm{A}_{n}$ in the cases that are of most interest for the Aschbacher-Scott program.

The paper is organized as follows. Basic notions are recalled in $\S 2$. Theorem 3.2 in $\S 3$ compares the dimensions of the Hom-spaces of irreducible $S_{n}$-modules in characteristic 2 over certain Young subgroups of $S_{n}$ when $n$ is even. Then Propositions 4.1, 4.3, and 4.4 in $\S 4$ show in particular that the $p$-modular irreducible representations of $\mathbf{A}_{n}$ which do not extend to $S_{n}$ must have large enough dimension (at least exponential in $n$ ). These results, which we believe are also of independent interest, allow us to discard non- $S_{n}$-extendible $\mathrm{A}_{n}$-modules in the proof of Theorem 1.2. In $\S 5$ we describe the submodule structure of the permutation modules of $\mathrm{S}_{n}$ acting on subsets of $\{1,2, \ldots, n\}$ of cardinality 2 or 3 in characteristic 2 , again in the case of even $n$. This description plays a key role in the proof of Theorem 6.5 in $\S 6$, which gives a criterion for a 2 -modular irreducible $\mathrm{S}_{n^{-}}$ representation to be reducible over certain subgroups of $S_{n}$. Theorem 6.5 is then used in $\S 7$ to show that non-trivial 2-modular irreducible $A_{n}$-representations are reducible over $\mathrm{A}_{m}$, if $\mathrm{A}_{m}$ is embedded into $\mathrm{A}_{n}$ via its actions on subsets or set partitions of $\{1,2, \ldots, m\}$ 
- see Theorem 7.12. Theorem 1.2 is proved in $\S 8$, which also contains further results concerning non-primitive embeddings of $A_{m}$ into $A_{n}$. The final $\S 9$ is devoted to the proof of Theorem 1.3.

\section{Preliminaries}

Throughout the paper, unless otherwise stated, we assume that the ground field $\mathbb{F}$ is algebraically closed, and $p:=\operatorname{char}(\mathbb{F})$. For a group $G$, the trivial $\mathbb{F} G$-module is denoted $\mathbf{1}_{G}$ or simply $\mathbf{1}$ if it is clear what $G$ is. If $V$ is an $\mathbb{F} G$-module, we denote by $\operatorname{soc}(V)$ the socle of $V$, and for $n=1,2, \ldots$, define $\operatorname{soc}^{n}(V)$ from $\operatorname{soc}^{1}(V)=\operatorname{soc}(V)$ and $\operatorname{soc}^{n}(V) / \operatorname{soc}^{n-1}(V)=\operatorname{soc}\left(V / \operatorname{soc}^{n-1}(V)\right)$ for $n>1$. We refer to the quotients $\operatorname{soc}^{n}(V) / \operatorname{soc}^{n-1}(V)$ as the socle layers of $V$ and usually list them from bottom to top, i.e. first $\operatorname{soc}(V)$, then $\operatorname{soc}^{2}(V) / \operatorname{soc}(V)$, etc.

For $n \in \mathbb{Z}_{>0}$, let

$$
\Omega:=\{1,2, \ldots, n\} .
$$

For $r=1, \ldots, n$, denote by $\Omega_{r}$ the set of $r$-element subsets of $\Omega$. The symmetric group $\mathrm{S}_{n}$ acts naturally on the sets $\Omega=\Omega_{1}, \Omega_{2}, \ldots, \Omega_{n}$ and the stabilizer of an element of $\Omega_{r}$ is conjugate to the subgroup $\mathrm{S}_{n-r, r}:=\mathrm{S}_{\{1,2, \ldots, n-r\}} \times \mathrm{S}_{\{n-r+1, \ldots, n\}}$. We write $\mathrm{S}_{n-1,1}$ simply as $\mathrm{S}_{n-1}$.

We denote by

$$
M_{r}=\mathbb{F} \Omega_{r} \cong \operatorname{Ind}_{\mathrm{S}_{n-r, r}}^{\mathrm{S}_{n}} \mathbf{1}_{\mathrm{S}_{n-r, r}} \quad(1 \leq r \leq n)
$$

the permutation module for the action of $\mathrm{S}_{n}$ on $\Omega_{r}$.

We recall some basic notions of representation theory of symmetric groups referring to [J2] for details. The irreducible $\mathbb{F} \mathrm{S}_{n}$-modules are labeled by $p$-regular partitions of $n$ (if $p=0$ then $p$-regular partitions are interpreted as all partitions). If $\lambda$ is a $p$-regular partition of $n$, the corresponding irreducible module is denoted $D^{\lambda}$. The Specht modules over $\mathbb{F S}_{n}$ are labeled by partitions of $n$. If $\lambda$ is such a partition, the corresponding Specht module is denoted $S^{\lambda}$.

Let $p=2$. Consider the partition

$$
\alpha_{n}= \begin{cases}(k+1, k-1) & \text { if } n=2 k \text { is even, } \\ (k+1, k) & \text { if } n=2 k+1 \text { is odd. }\end{cases}
$$

The irreducible module $D^{\alpha_{n}}$ is called the basic spin module for $\mathrm{S}_{n}$. It is known [W, Table III] that

$$
\operatorname{dim} D^{\alpha_{n}}=2^{\lfloor(n-1) / 2\rfloor} .
$$

Let $\operatorname{sgn}_{n}$ be the sign module over $\mathbb{F} S_{n}$. For any $p$-regular partition $\lambda$, we have that $D^{\lambda} \otimes \operatorname{sgn}_{n}$ is an irreducible $\mathbb{F} S_{n}$-module, so we can write $D^{\lambda} \otimes \operatorname{sgn}_{n} \cong D^{\lambda^{\mathrm{M}}}$, where

$$
\mathbf{M}: \lambda \mapsto \lambda^{\mathbf{M}}
$$

is the Mullineux involution on the set of $p$-regular partitions of $n$. To describe the Mullineux involution, we briefly recall the notion of the Mullineux symbol $G(\lambda)$ of $\lambda$, referring the reader to $[\mathrm{FK}]$ for details. Let $h_{1}$ be the number of nodes in the $p$-rim of $\lambda$, 
and let $r_{1}$ be the number of rows in $\lambda$. Delete the $p$-rim and repeat to obtain sequences $h_{1}, h_{2}, \ldots$ and $r_{1}, r_{2}, \ldots$ Let $k$ be such that $h_{k+1}=r_{k+1}=0$ but $h_{k} \neq 0 \neq r_{k}$. Then

$$
G(\lambda):=\left(\begin{array}{cccc}
h_{1} & h_{2} & \ldots & h_{k} \\
r_{1} & r_{2} & \ldots & r_{k}
\end{array}\right) .
$$

It was proved in $[\mathrm{Mu}]$ that $\lambda$ is uniquely determined by $G(\lambda)$. Moreover, we have

$$
G\left(\lambda^{\mathbf{M}}\right)=\left(\begin{array}{cccc}
h_{1} & h_{2} & \ldots & h_{k} \\
h_{1}-r_{1}+\epsilon_{1} & h_{2}-r_{2}+\epsilon_{2} & \ldots & h_{k}-r_{k}+\epsilon_{k}
\end{array}\right),
$$

where $\epsilon_{i}:=0$ if $p \mid h_{i}$ and $\epsilon_{i}:=1$ otherwise. This description of $\mathbf{M}$ is the main result of [FK] (see also $[\mathrm{BeO}]$ ), which was conjectured by Mullineux.

Given an irreducible representation $D^{\lambda}$, either the restriction $E^{\lambda}:=\operatorname{Res}_{\mathrm{A}_{n}}^{S_{n}} D^{\lambda}$ is irreducible or $\operatorname{Res}_{\mathrm{A}_{n}}^{\mathrm{S}_{n}} D^{\lambda} \cong E_{+}^{\lambda} \oplus E_{-}^{\lambda}$, a direct sum of two inequivalent irreducible representations. Moreover, every irreducible $\mathbb{F} \mathrm{A}_{n}$-module is isomorphic to one of $E_{( \pm)}^{\lambda}$, and the only non-trivial isomorphism of the form $E_{( \pm)}^{\lambda} \cong E_{( \pm)}^{\mu}$ is $E^{\lambda} \cong E^{\lambda^{\mathrm{M}}}$.

If $p \neq 2$, then $\operatorname{Res}_{\mathbf{A}_{n}}^{\mathbf{S}_{n}} D^{\lambda}$ is reducible if and only if $\lambda=\lambda^{\mathbf{M}}$. If $p=2$, then an explicit criterion for reducibility of $\operatorname{Res}_{\mathrm{A}_{n}}^{\mathrm{S}_{n}} D^{\lambda}$ is given in [Ben, Theorem 1.1].

\section{Comparing some Hom-SpaCes}

Throughout this section we assume that $p=2$. In this section we get some results on the dimensions

$$
d_{r}(V):=\operatorname{dim} \operatorname{Hom}_{\mathbb{F} \mathbf{S}_{n}}\left(M_{r}, \operatorname{End}_{\mathbb{F}}(V)\right)=\operatorname{dim} \operatorname{End}_{\mathbb{F} \mathbf{S}_{n-r, r}}\left(\operatorname{Res}_{\mathbf{S}_{n-r, r}}^{\mathbf{S}_{n}} V\right) .
$$

The last equality follows using $M_{r}=\operatorname{Ind}_{\mathrm{S}_{n-r, r}}^{\mathrm{S}_{n}} \mathbf{1}_{\mathrm{S}_{n-r, r}}$ and Frobenius reciprocity.

Lemma 3.1. Let $V$ be an irreducible $\mathbb{F S}_{n}$-module and $1 \leq r \leq n$. Then $d_{r}(V)=1$ if and only if $\operatorname{Res}_{\mathrm{S}_{n-r, r}} V$ is irreducible.

Proof. The sufficiency of the condition is clear. Conversely, irreducible $\mathbb{F} \mathrm{S}_{n}$-modules are self-dual, so the restriction $\operatorname{Res}_{\mathrm{S}_{n-r, r}}^{\mathrm{S}_{n}} V$ is self-dual. Since irreducible $\mathbb{F S}_{n-r, r}$-modules are also self-dual, the head of $\operatorname{Res}_{\mathbf{S}_{n-r, r}}^{\mathbf{S}_{n}} V$ is isomorphic to its socle. So if $\operatorname{Res}_{\mathbf{S}_{n-r, r}} V$ is reducible then $d_{r}(V)>1$.

The goal of this section is to prove the following result:

Theorem 3.2. Let $V$ be a simple $\mathbb{F} S_{n}$-module and $2 \mid n \geq 6$. Then one of the following statements holds:

(i) $d_{3}(V)>d_{1}(V)$.

(ii) $V \cong D^{\alpha_{n}}$ is the basic spin module or $V \cong \mathbf{1}$ is the trivial module, in which cases we have $d_{3}(V)=d_{1}(V)$.

Let $V=D^{\lambda}$ for a 2-regular partition

$$
\lambda=\left(\lambda_{1}>\ldots>\lambda_{s}>0\right)
$$

of $n$. If $s=1$, then $V$ is the trivial module, and Theorem 3.2 holds trivially. 
Lemma 3.3. Theorem 3.2 holds if $d_{1}(V)=1$.

Proof. Suppose that $d_{1}(V)=d_{3}(V)=1$ and $\operatorname{dim} V>1$. By Lemma 3.1, $V$ is irreducible over $S_{n-1}$ and $S_{3, n-3}$, and so the lemma follows from [P, Theorem 10].

Lemma 3.4. Theorem 3.2 holds if $s=2$.

Proof. Since $n$ is even, we have that the restriction $D^{\lambda} \mid \mathrm{S}_{n-1}$ is irreducible by [K1]. So $d_{1}(V)=1$, and we may apply Lemma 3.3.

Lemma 3.5. Let

$$
x:=\sum_{g \in \mathrm{S}_{\{1,2,3\}},} \sum_{\sigma \in \mathrm{S}_{\{1,4\}} \times \mathrm{S}_{\{2,5\}} \times \mathrm{S}_{\{3,6\}}}(\operatorname{sign} \sigma) \sigma g \sigma^{-1} \in \mathbb{F S}_{n} .
$$

If $s>2$, then $x D^{\lambda} \neq 0$.

Proof. By [BaK, Lemma 4.7] with $(m, p)=(3,2)$, we see that the restriction $\left.V\right|_{\mathrm{s}_{6}}$ has a composition factor isomorphic to $D^{(3,2,1)}$, which for $p=2$ is isomorphic to the Specht module $S^{(3,2,1)}$. Since $x \in \mathbb{F S}_{6}$, it suffices to prove that $x S^{(3,2,1)} \neq 0$. We use the notation of $[\mathrm{J} 2, \S 4]$; in particular, $e_{t}$ is the polytabloid and $\{t\}$ is the tabloid corresponding to a $(3,2,1)$-tableau $t$. Let

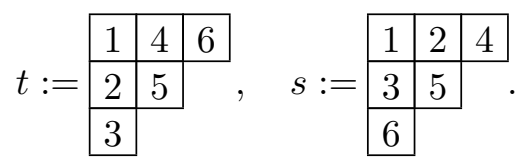

An explicit calculation shows that $\{s\}$ appears in $x e_{t}$ with coefficient 1 .

We now complete the proof of Theorem 3.2. Recall that $M_{3}$ is the permutation module on all three element subsets $\{i, j, k\} \subseteq \Omega$, while $M_{1}$ is the permutation module on the one element subsets $\{i\} \subseteq \Omega$. Consider the $\mathbb{F S}_{n}$-module homomorphism

$$
f: M_{3} \rightarrow M_{1},\{i, j, k\} \mapsto\{i\}+\{j\}+\{k\} .
$$

It is easy to see that $f$ is surjective. So it induces an injective linear map

$$
f^{*}: \operatorname{Hom}_{\mathbb{F} S_{n}}\left(M_{1}, \operatorname{End}_{\mathbb{F}}(V)\right) \rightarrow \operatorname{Hom}_{\mathbb{F} S_{n}}\left(M_{3}, \operatorname{End}_{\mathbb{F}}(V)\right), \psi \mapsto \psi \circ f .
$$

It suffices to prove that $f^{*}$ is not surjective.

We exhibit an element $\phi \in \operatorname{Hom}_{\mathbb{F} S_{n}}\left(M_{3}, \operatorname{End}_{\mathbb{F}}(V)\right)$ which is not in the image of $f^{*}$. For $\Theta \subseteq \Omega$ let $\mathrm{S}_{\Theta} \subseteq \mathrm{S}_{n}=\mathrm{S}_{\Omega}$ be the subgroup of all permutations which stabilize the elements of $\Omega \backslash \Theta$. Now, define $\phi$ as follows:

$$
\phi(\{i, j, k\})(v):=\sum_{g \in \mathrm{S}_{\{i, j, k\}}} g v, \quad(\{i, j, k\} \subseteq \Omega, v \in V) .
$$

If $\phi \in \operatorname{Im} f^{*}$, then $\phi=\psi \circ f$ for some $\psi \in \operatorname{Hom}_{\mathbb{F} S_{n}}\left(M_{1}, \operatorname{End}_{\mathbb{F}}(V)\right)$. Consider the element

$$
E=\sum_{\sigma \in \mathrm{S}_{\{1,4\}} \times \mathrm{S}_{\{2,5\}} \times \mathrm{S}_{\{3,6\}}}(\operatorname{sign} \sigma) \sigma\{1,2,3\} \in M_{3} .
$$


Note that $f(E)=0$. So $\phi(E)=\psi(f(E))=0$. On the other hand, we compute $\phi(E)$ using (3.2):

$$
\phi(E)(v)=\sum_{\sigma \in \mathrm{S}_{\{1,4\}} \times \mathrm{S}_{\{2,5\}} \times \mathrm{S}_{\{3,6\}}(\operatorname{sign} \sigma)} \sum_{g \in \mathrm{S}_{\{\sigma(1), \sigma(2), \sigma(3)\}}} g v=x v,
$$

where $x$ is as in Lemma 3.5. Now Lemma 3.5 yields a contradiction.

\section{Dimension and extendibility to $\mathrm{S}_{n}$}

First we prove the following statement, which relies on some results of [Ben] and [GLT]:

Proposition 4.1. Let $p=2, n \geq 5$, and let $V$ be an irreducible $\mathbb{F} \mathrm{A}_{n}$-module. Suppose that $V$ does not extend to $\mathrm{S}_{n}$. Then $\operatorname{dim} V \geq 2^{(n-6) / 4}$.

Proof. By assumption, $W:=\operatorname{Ind}_{\mathrm{A}_{n}}^{\mathrm{S}_{n}}(V)$ is an irreducible $\mathbb{F S}_{n}$-module, and $\operatorname{dim} W=$ $2 \operatorname{dim} V$. Let $\lambda=\left(\lambda_{1}>\lambda_{2}>\ldots \lambda_{s}>0\right)$ be the partition of $n$ into distinct parts corresponding to $W$. Since $W$ is reducible over $\mathrm{A}_{n}$, by [Ben, Theorem 1.1], we have $s>1$ and $\lambda_{1}-\lambda_{2} \in\{1,2\}$. In particular, $n \geq \lambda_{1}+\lambda_{2} \geq 2 \lambda_{1}-2$, i.e. $\lambda_{1} \leq(n+2) / 2$. Now

$$
\operatorname{dim} W \geq 2^{\frac{n-\lambda_{1}}{2}} \geq 2^{\frac{n-(n+2) / 2}{2}}=2^{\frac{n-2}{4}},
$$

thanks to [GLT, Theorem 5.1].

We will also need the following branching result which is of interest in its own right:

Proposition 4.2. Let $p=2$, and $\lambda=\left(\lambda_{1}>\ldots>\lambda_{s}>0\right) \neq(n)$ be a non-trivial 2-regular partition of $n$. If $2 \lambda_{1}-n \geq k \geq 3$ then the restriction of $D^{\lambda}$ to a natural subgroup $\mathrm{S}_{k}$ of $\mathrm{S}_{n}$ affords both $\mathbf{1}=D^{(\bar{k})}$ and $D^{(k-1,1)}$ as composition factors.

Proof. We apply induction on $m:=n-\lambda_{1} \geq 1$. If $m=1$, then $D^{\lambda}=D^{(n-1,1)}$ is the heart of the natural permutation module, and the statement follows easily. Let $m \geq 2$.

Case 1: $\lambda_{1}-\lambda_{2}$ is odd. Then, in the terminology of [K2, Definition 0.3], 2 is a normal index. Let $j \geq 2$ be the largest normal index; in particular $j$ is a good index in the sense of [K2, Definition 0.3] again. Then by [K2, Theorem 0.5], $D^{\mu}$ is a simple submodule of $\left.D^{\lambda}\right|_{\mathrm{S}_{n-1}}$, where

$$
\mu=\lambda(j):=\left(\lambda_{1}, \ldots, \lambda_{j-1}, \lambda_{j}-1, \lambda_{j+1}, \ldots, \lambda_{s}\right) \vdash(n-1) .
$$

Note that $2 \lambda_{1}-(n-1) \geq k+1$ and $(n-1)-\lambda_{1}=m-1$. Hence we can apply the induction hypothesis to $D^{\mu}$ restricted to $\mathrm{S}_{k}$.

Case 2: $\lambda_{1}-\lambda_{2}$ is even. Now 1 is a normal index. Then by [K2, Theorem 0.4], $D^{\nu}$ is a composition factor of $D^{\lambda} \mid \mathrm{s}_{n-1}$, where

$$
\nu=\lambda(1):=\left(\lambda_{1}-1, \lambda_{2}, \ldots, \lambda_{s}\right) \vdash(n-1) .
$$

Since $\left(\lambda_{1}-1\right)-\lambda_{2}$ is odd, as in Case 1 we now see that 2 is a normal index for $\nu$. Let $j \geq 2$ be the highest normal index of $\nu$; in particular $j$ is a good index. Then again by $\left[\mathrm{K} 2\right.$, Theorem 0.5], $D^{\mu}$ is a simple submodule of $D^{\lambda} \mid \mathrm{s}_{n-2}$, where

$$
\mu=\nu(j):=\left(\lambda_{1}-1, \ldots, \lambda_{j-1}, \lambda_{j}-1, \lambda_{j+1}, \ldots, \lambda_{s}\right) \vdash(n-2) .
$$


Note that $2\left(\lambda_{1}-1\right)-(n-2) \geq k$ and $(n-2)-\left(\lambda_{1}-1\right)=m-1$. Hence we can apply the induction hypothesis to $D^{\mu}$ restricted to $\mathrm{S}_{k}$.

Using the Mullineux involution, we prove an analogue of Proposition 4.1 for $p \neq 2$ (certainly, the most interesting case being $p \leq n$ ):

Proposition 4.3. Let $n \geq 5$ and $p \neq 2$.

(i) Let $\lambda=\left(\lambda_{1}, \lambda_{2}, \ldots\right)$ be a p-regular partition of $n$. Suppose that $\lambda_{1} \geq(n+p+2) / 2$. Then $D^{\lambda}$ is irreducible over $\mathrm{A}_{n}$.

(ii) Let $V$ be an irreducible $\mathbb{F} \mathrm{A}_{n}$-module. Suppose that $V$ does not extend to $\mathrm{S}_{n}$. Then $\operatorname{dim} V \geq 2^{(n-p-5) / 4}$.

Proof. (i) Recalling the definition of the Mullineux map from Section 2, denote the partitions obtained from $\lambda$ by successively removing $p$-rims as $\lambda^{(j)}, 1 \leq j \leq k$. We prove the statement by induction on $n-\lambda_{1}$. Since $\lambda_{1} \geq\left(n-\lambda_{1}\right)+p+2$ by the assumption, the first $p$-segment of the $p$-rim of $\lambda$ has length $p$. Assume for a contradiction that $\lambda=\lambda^{\mathbf{M}}$. Then

$$
h_{i}+\epsilon_{i}=2 r_{i}
$$

for $1 \leq i \leq k$.

Suppose first that the $p$-rim of $\lambda^{\prime}:=\left(\lambda_{2}, \lambda_{3}, \ldots\right)$ has at most $p-1$ nodes. Write $h_{1}=p+x$ and $r_{1}=1+y$, where $0 \leq x \leq p-1$ and $y$ is the number of rows of $\lambda^{\prime}$. Then according to (4.1) we have

$$
p+x \leq p+x+\epsilon_{1}=h_{1}+\epsilon_{1}=2 r_{1}=2+2 y .
$$

Note that $x$ is the length of the $p$-rim of $\lambda^{\prime}$. Hence $y \leq x$, and so (4.2) yields $x \geq p-2>0$. In turn, this implies that $p \nmid h_{1}$, whence $\epsilon_{1}=1$ and (4.2) yields $x=y=p-1$ (as $\left.x \leq p-1\right)$. Recall we are assuming that the $p$-rim of $\lambda^{\prime}$ has at most $p-1$ nodes. It follows that the $p$-rim of $\lambda^{\prime}$ has exactly $p-1$ nodes and $\lambda^{\prime}$ also has $p-1$ rows. This can happen only when $\lambda^{\prime}=\left(1^{p-1}\right)$, a column of $p-1$ nodes. In this case, $\lambda^{(1)}=\left(\lambda_{1}-p\right)$ has one part, which is of length $\geq 2$. Hence the $p$-rim of $\lambda^{(1)}$ is of length $p$ (if $\lambda_{1} \geq 2 p$ ), or $z \geq 2$ (where $2 p-1 \geq \lambda_{1}=p+z \geq p+2$ ). Correspondingly, $r_{2}=1$ and $\left(h_{2}, \epsilon_{2}\right)=(p, 0)$ or $(z, 1)$. In either case

$$
h_{2}+\epsilon_{2} \geq z+1 \geq 3>2 r_{2},
$$

contrary to (4.1).

Assume now that the $p$-rim of $\lambda^{\prime}$ has at least $p$ nodes. Then, aside from the first $p$-segment contained in the first row, the $p$-rim of $\lambda$ contains at least $p$ nodes of $\lambda^{\prime}$. It follows that the condition $\lambda_{1} \geq\left(n-\lambda_{1}\right)+p+2$ also holds for $\lambda^{(1)}$. By the induction hypothesis, $\lambda^{(1)}$ is not equal to its Mullineux dual, i.e. $h_{i}-r_{i}+\epsilon_{i} \neq r_{i}$ for some $i \geq 2$, again contradicting (4.1).

(ii) By assumption, $W:=\operatorname{Ind}_{\mathrm{A}_{n}}^{\mathrm{S}_{n}}(V)$ is an irreducible $\mathbb{F S} \mathrm{S}_{n}$-module and $\operatorname{dim} W=$ $2 \operatorname{dim} V$. Let $\lambda=\left(\lambda_{1}, \lambda_{2}, \ldots\right)$ be the $p$-regular partition of $n$ corresponding to $W$. Since $W$ is reducible over $\mathrm{A}_{n}, \lambda_{1} \leq(n+p+1) / 2$ by (i). It now follows by [GLT, Theorem 5.1] that

$$
\operatorname{dim} W \geq 2^{\frac{n-\lambda_{1}}{2}} \geq 2^{\frac{n-(n+p+1) / 2}{2}}=2^{\frac{n-p-1}{4}},
$$


which implies the result.

Here is another version of Proposition 4.3:

Proposition 4.4. Let $p>2, n \geq 5$, and $\lambda=\left(\lambda_{1}, \lambda_{2}, \ldots\right)$ be a p-regular partition of $n$. Suppose that there is some $s \geq 1$ such that

$$
\lambda_{1}-\lambda_{2} \geq \lambda_{2}-\lambda_{3} \geq \ldots \geq \lambda_{s}-\lambda_{s+1} \geq p
$$

and

$$
\sum_{i=1}^{s}\left\lfloor\frac{\lambda_{i}-\lambda_{i+1}}{p}\right\rfloor>\frac{n}{2 p-1} .
$$

Then $D^{\lambda}$ is irreducible over $\mathrm{A}_{n}$. In particular, if

$$
\lambda_{1} \geq \lambda_{2}+p\left\lceil\frac{n+1}{2 p-1}\right\rceil
$$

then $D^{\lambda}$ is irreducible over $\mathrm{A}_{n}$.

Proof. Assume that $D^{\lambda}$ is reducible over $\mathrm{A}_{n}$. Then $\lambda=\lambda^{\mathbf{M}}$ and so

$$
\sum_{i=1}^{k}\left(h_{i}-2 r_{i}+\epsilon_{i}\right)=0 .
$$

We will estimate $h_{1}-2 r_{1}+\epsilon_{1}$ by going down the $p$-segments of the $p$-rim of $\lambda$. Since $\lambda_{1}-\lambda_{2} \geq p$, the first $p$-segment consists of $p$ nodes of the first row and so contributes $p-2$ to $h_{1}-2 r_{1}+\epsilon_{1}$. More generally, any horizontal $p$-segment of length $p$ contributes $p-2$ to $h_{1}-2 r_{1}+\epsilon_{1}$. On the other hand, since $\lambda$ is $p$-regular, any non-horizontal $p$-segment of length $p$ has height $\leq(p-1)$ and so it contributes at least $p-2(p-1)=2-p$ to $h_{1}-2 r_{1}+\epsilon_{1}$. Suppose the $p$-rim also has a $p$-segment of length $j$ less than $p$. Then it must be the last $p$-segment, and $\epsilon_{1}=1$. So the contribution of this $p$-segment to $h_{1}-2 r_{1}+\epsilon_{1}$ is $\geq j-2 j+1=1-j$.

As the $p$-rims are removed in succession, let $a$ be the total number of horizontal $p$ segments of length $p, b_{p}$ be the total number of non-horizontal $p$-segments of length $p$, and $b_{j}$ be the total number of $p$-segments of length $1 \leq j<p$, so that $n=p a+\sum_{j=1}^{p} j b_{j}$. Applying the above arguments to all successive $p$-rims of $\lambda$ we have that

$$
\begin{aligned}
\sum_{i=1}^{k}\left(h_{i}-2 r_{i}+\epsilon_{i}\right) & \geq(p-2) a-(p-2) b_{p}-\sum_{j=1}^{p-1}(j-1) b_{j} \\
& \geq(2 p-2) a+\left(2 b_{p}+\sum_{j=1}^{p-1} b_{j}\right)-\left(p a+\sum_{j=1}^{p} j b_{j}\right) \\
& \geq(2 p-2) a+\left(2 b_{p}+\sum_{j=1}^{p-1} b_{j}\right)-n .
\end{aligned}
$$


Observe that

$$
2 b_{p}+\sum_{j=1}^{p-1} b_{j} \geq \frac{\sum_{j=1}^{p} j b_{j}}{p-1}=\frac{n-p a}{p-1} .
$$

Under the hypothesis, we can find an integer $t \geq(n+1) /(2 p-1)$ such that

$$
\sum_{i=1}^{s}\left\lfloor\frac{\lambda_{i}-\lambda_{i+1}}{p}\right\rfloor \geq t
$$

Now observe that at least $t$ horizontal $p$-segments from the first $s$ rows of $\lambda$ belong to these successive $p$-rims. Thus $a \geq t \geq(n+1) /(2 p-1)$. Hence,

$$
\sum_{i=1}^{k}\left(h_{i}-2 r_{i}+\epsilon_{i}\right) \geq(2 p-2) a+\frac{n-p a}{p-1}-n \geq \frac{p-2}{p-1}((2 p-1) a-n)>0,
$$

contradicting (4.3).

\section{Structure of Permutation modules}

Throughout the section $n \geq 6$ is an even integer and $p=2$.

We will study permutation modules $M_{r}$, mainly for $r=1,2,3$. For $n \geq 2 r$, let $\mathcal{S}_{r} \subset M_{r}$ denote the Specht module $S^{(n-r, r)}$ and (assuming $n>2 r$ ) let $D_{r}=D^{(n-r, r)}$ be the unique simple quotient of $\mathcal{S}_{r}$. Let $T_{r} \in M_{r}$ be the sum of all $r$-element sets. Let

$$
\eta_{r, s}: M_{r} \rightarrow M_{s}
$$

denote the incidence homomorphism sending an $r$-set to the sum of $s$-sets incident with (i.e. containing or contained in) it. By [J2, Corollary 17.18],

$$
\mathcal{S}_{r}=\cap_{t=0}^{r-1} \operatorname{Ker} \eta_{r, t} .
$$

We denote by $M_{r}^{\prime}$ the augmentation module, i.e. the submodule Ker $\eta_{r, 0}$ of $M_{r}$ (spanned by differences of pairs of basis elements).

The space $M_{r}$ has a natural bilinear form $\langle\cdot, \cdot\rangle_{r}$, with respect to which the standard basis is orthonormal. If we identify $M_{r}$ and $M_{s}$ with their dual spaces, using the corresponding bilinear forms, then $\eta_{s, r}$ is the dual map of $\eta_{r, s}$. In particular, $\eta_{s, r}$ is injective iff $\eta_{r, s}$ is surjective and vice versa. Also $\operatorname{Im} \eta_{s, r} \cong \operatorname{Im} \eta_{r, s}^{*}$ as $\mathbb{F S} n_{n}$-modules. We have

$$
\left\langle x, \eta_{r, s}(y)\right\rangle_{s}=\left\langle\eta_{s, r}(x), y\right\rangle_{r} \quad\left(x \in M_{s}, y \in M_{r}\right) .
$$

The ranks of the maps $\eta_{r, s}$ are given in [Wil]. We state the special cases that we need of this general result.

Lemma 5.1. For $r \leq \min \{s, n-s\}$ we have

$$
\operatorname{rank}_{\mathbb{F}} \eta_{r, s}=\sum_{1 \leq i \leq r,}\left(\left(\begin{array}{c}
n \\
i
\end{array}\right)-\left(\begin{array}{c}
n \\
i-i \\
i-1
\end{array}\right)\right) \text { is odd }
$$

In particular, $\eta_{1,3}$ is injective, and

$$
\operatorname{rank}_{\mathbb{F}} \eta_{1,2}=n-1=\operatorname{dim} \mathcal{S}_{1}, \quad \operatorname{rank}_{\mathbb{F}} \eta_{2,3}=1+\frac{n(n-3)}{2}=1+\operatorname{dim} \mathcal{S}_{2} .
$$


Lemma 5.2. $M_{1}$ is a uniserial $\mathbb{F} S_{n}$-module with socle layers $\mathbb{F} \cdot T_{1} \cong \mathbf{1}, D_{1}, \mathbf{1}$.

Proof. This is well known, see e.g. [J2, Example 5.1].

Let the $\mathbb{F S} S_{n}$-module $Q$ be defined by the short exact sequence

$$
0 \rightarrow \mathbb{F} \cdot T_{1} \rightarrow M_{1} \rightarrow Q \rightarrow 0 .
$$

In fact, $Q \cong \mathcal{S}_{1}^{*}$.

The following lemma can be deduced from [MO, Theorem (1.1)], but we give an independent proof for the reader's convenience:

Lemma 5.3. As $\mathbb{F} S_{n}$-modules, $\operatorname{Im} \eta_{1,2} \cong Q$ and $M_{2}$ has the following structure:

(i) If $n \equiv 0(\bmod 4)$ then the composition factors of $M_{2}$ are $\mathbf{1}$ (twice), $D_{1}$ (twice) and $D_{2}$ (once). Furthermore, $M_{2} /\left(\operatorname{Im} \eta_{1,2}+\mathbb{F} \cdot T_{2}\right) \cong S_{2}^{*}$, whose socle is $D_{2}$.

(ii) If $n \equiv 2(\bmod 4)$ then $M_{2} \cong \mathbb{F} \cdot T_{2} \oplus M_{2}^{\prime}$, and $M_{2}^{\prime}$ is uniserial with socle layers $D_{1}, 1, D_{2}, 1, D_{1}$.

Proof. The dimension of $\operatorname{Im} \eta_{1,2}$ is given by Lemma 5.1, from which we see that it is isomorphic to $Q$. The composition factors are given by [J1].

(i) The second statement in (i) now follows using the facts that the dual Specht module $S_{2}^{*}$ is a quotient of $M_{2}$ and $S_{2}^{*}$ is uniserial with socle layers $D_{2}, D_{1}$.

(ii) The submodule $\mathbb{F} \cdot T_{2}$ is a direct summand of $M_{2}$ because $\left(\begin{array}{l}n \\ 2\end{array}\right)$ is odd. Then $M_{2}^{\prime}=\operatorname{Ker} \eta_{2,0}$ is the complementary summand. The composition factors of $M_{2}^{\prime}$ are $D_{1}$, $\mathbf{1}, D_{2}, \mathbf{1}, D_{1}$. Also we have $\mathcal{S}_{2} \subseteq M_{2}^{\prime}$. The composition factors of $\mathcal{S}_{2}$ are $D_{2}, D_{1}, \mathbf{1}$ and $\mathcal{S}_{2}$ has a simple head isomorphic to $D_{2}$. Since $M_{2}^{\prime \mathrm{S}_{n}}=0$, it follows that $\mathcal{S}_{2}$ must be uniserial with socle layers $D_{1}, \mathbf{1}, D_{2}$. The uniseriality of $M_{2}^{\prime}$ and its socle layers now follow from the self-duality of $M_{2}^{\prime}$.

Lemma 5.4. The $\mathbb{F S}{ }_{n}$-module $M_{3}$ has the following structure.

(i) If $n \equiv 0(\bmod 4)$, then $M_{3} \cong M_{1} \oplus U$, where $U$ is uniserial with socle layers $D_{2}$, $D_{1}, D_{3}, D_{1}, D_{2}$.

(ii) If $n \equiv 2(\bmod 4)$ then $\operatorname{Im} \eta_{2,3}$ is uniserial with socle layers $\mathbf{1}, D_{2}, \mathbf{1}, D_{1}$. The composition factors of $M_{3}$ are $\mathbf{1}$ (with multiplicity 4), $D_{1}$ (twice), $D_{2}$ (twice) and $D_{3}$ (once). Also, $\operatorname{soc}\left(M_{3}\right)=\mathbb{F} \cdot T_{3}$.

Proof. The composition factors are given by [J1]. The dimensions of the images of the incidence maps $\eta_{r, s}$ are given by Lemma 5.1 .

(i) A simple computation shows that $\eta_{3,1} \circ \eta_{1,3}=1_{M_{1}}$, so $\eta_{1,3}$ is a split injection of $M_{1}$ into $M_{3}$. In fact, by computing bilinear forms on the images of basis elements, $\eta_{1,3}$ is seen to be an isometry. So $M_{3}=\operatorname{Im} \eta_{1,3} \oplus U$, with $U=\left(\operatorname{Im} \eta_{1,3}\right)^{\perp}=\operatorname{Ker} \eta_{3,1}$, where the last equality is by (5.2). The module $U$ is a self-dual module and its composition factors are $D_{1}$ (twice), $D_{2}$ (twice) and $D_{3}$, and $U$ contains $\mathcal{S}_{3}$. The structure of $U$ will follow from its self-duality if we prove that $\mathcal{S}_{3}$ is uniserial with socle layers $D_{2}, D_{1}, D_{3}$. Since we know that the head of $\mathcal{S}_{3}$ is isomorphic to $D_{3}$, it suffices to show that $D_{1}$ is not a submodule of $\mathcal{S}_{3}$. If it were, then we would have $\operatorname{Hom}_{\mathbb{F} S_{n}}\left(D_{1}, M_{3}\right) \neq 0$, whence $\operatorname{Hom}_{\mathbb{F} \mathrm{S}_{n}}\left(M_{3}, D_{1}\right) \neq 0$, i.e. the fixed point subspace $D_{1}^{\mathrm{S}_{n-3,3}}$ is non-trivial, which is easily checked to be false. 
(ii) We have $\operatorname{Im} \eta_{1,2} \cong Q$. Also, it is easy to see that $\eta_{2,3} \circ \eta_{1,2}=0$, whence by dimensions using Lemma 5.1, we conclude that $\operatorname{Im} \eta_{1,2}=\operatorname{Ker} \eta_{2,3}$. Moreover, $\operatorname{Im} \eta_{1,2} \cong Q$ by Lemma 5.3. Since $\operatorname{Im} \eta_{1,2} \nsubseteq M_{2}^{\prime}$, the structure of $Q$ implies that $\operatorname{Ker} \eta_{2,3} \cap M_{2}^{\prime}$ must be zero or isomorphic to $D_{1}$. Since we know the rank of $\eta_{2,3}$, we see that the latter holds. Thus from the structure of $M_{2}^{\prime}$, we see that $\eta_{2,3}\left(M_{2}^{\prime}\right) \cong M_{2}^{\prime} / \operatorname{soc}\left(M_{2}^{\prime}\right)$. By dimensions, we see that $\eta_{2,3}\left(M_{2}^{\prime}\right)=\operatorname{Im} \eta_{2,3}$, so $\operatorname{Im} \eta_{2,3}$ is uniserial with the socle layers as stated.

It remains to show that $\operatorname{soc}\left(M_{3}\right)=\mathbb{F} \cdot T_{3}$. For this, it suffices to prove that $M_{3}$ has no submodule isomorphic to $D_{1}, D_{2}$ or $D_{3}$. For $D_{1}$ we explicitly check as in (i) that $D_{1}^{\mathrm{S}_{n-3,3}}=0$. The unique $D_{3}$ composition factor of $M_{3}$ is the head of $\mathcal{S}_{3}$, so since $\mathcal{S}_{3}$ is not simple, it follows that $M_{3}$ has no submodule isomorphic to $D_{3}$. Finally, we consider $D_{2}$. By the first part of (ii), Im $\eta_{2,3}$ has one composition factor $D_{2}$ as its second socle layer, so it suffices to show that $M_{3} / \operatorname{Im} \eta_{2,3}$, which has a single $D_{2}$ composition factor, has no submodule isomorphic to $D_{2}$. However, $M_{3} / \operatorname{Im} \eta_{2,3}$ has $D_{3}$ as a composition factor, so maps surjectively onto $\mathcal{S}_{3}^{*}$. By [J1], $D_{2}$ is a composition factor of $\mathcal{S}_{3}^{*}$, but $\mathcal{S}_{3}^{*}$ has a simple socle isomorphic to $D_{3}$. So $D_{2}$ is not a submodule of $M_{3}$.

Figures 1 and 2 below are given for the reader's convenience, but they will not be used in proofs. The pictures give partial information on submodule structure of the permutation modules $M_{2}$ and $M_{3}$. The edges indicate the existence of uniserial subquotients.

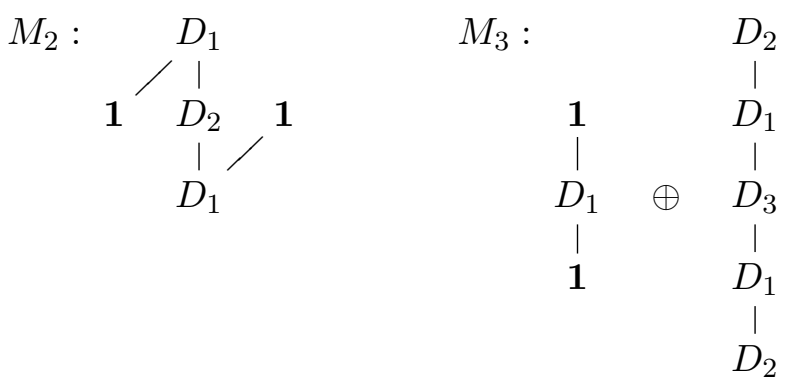

FiguRE 1. Submodule structures for $n \equiv 0(\bmod 4)$

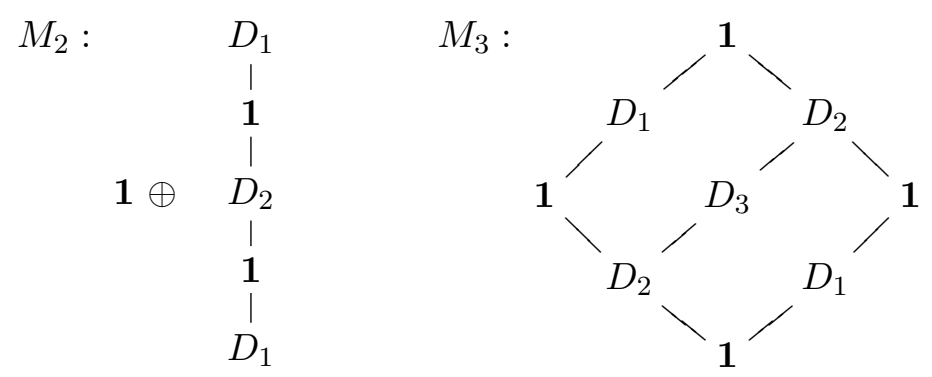

Figure 2. Submodule structures for $n \equiv 2(\bmod 4)$

Lemma 5.5. We have: 
(i) $\operatorname{Im} \eta_{1,3}$ is the unique submodule of $M_{3}$ that is isomorphic to $M_{1}$ as $\mathbb{F S}_{n}$-modules.

(ii) Ker $\eta_{3,1}$ is the unique submodule $N$ of $M_{3}$ such that $M_{3} / N \cong M_{1}$ as $\mathbb{F S}_{n}$-modules.

Proof. Part (ii) follows from (i) by the duality of $\eta_{1,3}$ and $\eta_{3,1}$. For (i), note that $\operatorname{dim} \operatorname{Hom}_{\mathbb{F S}}\left(M_{1}, M_{3}\right)=2$. The map $\eta_{1,3}$ and the map $\beta$ sending each 1-set to $T_{3}$ form a basis of this Hom-space. Now $\eta_{1,3}$ is injective since $\operatorname{soc}\left(M_{1}\right)$ is spanned by $T_{1}$ and $\eta_{1,3}\left(T_{1}\right)=3 T_{3}=T_{3} \neq 0$. Also we have $\operatorname{Im} \beta \subset \operatorname{Im} \eta_{1,3}$, so $\operatorname{Im} \eta_{1,3}$ is the unique submodule of $M_{3}$ isomorphic to $M_{1}$.

In the following two lemmas, $N$ denotes the submodule of $M_{3}$ specified in Lemma 5.5(ii).

Lemma 5.6. Let $n \equiv 0(\bmod 4)$. Then $\operatorname{Ker} \eta_{3,2} \cap N=\operatorname{soc}^{3}(N)$, and

$$
N / \operatorname{soc}^{3}(N) \cong \eta_{3,2}(N)=\operatorname{Im} \eta_{3,2} \cap M_{2}^{\prime}=\mathcal{S}_{2}
$$

Proof. By Lemma 5.4, N/ $\operatorname{soc}^{3}(N)$ is uniserial with socle $D_{1}$ and head $D_{2}$. Since $M_{2}$ has no composition factor isomorphic to $D_{3}$, we have $\operatorname{soc}^{3}(N) \subseteq \operatorname{Ker} \eta_{3,2}$.

We claim that the induced map $N / \operatorname{soc}^{3}(N) \rightarrow M_{2}$ is injective. If not, its image is either zero or isomorphic to $D_{2}$. The latter is impossible since $M_{2}$ has no submodule isomorphic to $D_{2}$. The former is also impossible since it forces the rank of $\eta_{3,2}$ to be at most $\operatorname{dim} M_{1}=n$, contrary to Lemma 5.1, which gives the actual rank as $1+\operatorname{dim} \mathcal{S}_{2}$.

Thus the map $\eta_{3,2}$ induces an isomorphism of $N / \operatorname{soc}^{3}(N)$ with a submodule $\eta_{3,2}(N) \subseteq$ $M_{2}$. Since $N \subseteq M_{3}^{\prime}$ and $\eta_{2,0} \circ \eta_{3,2}=\eta_{3,0}$, we have $\eta_{3,2}(N) \subseteq M_{2}^{\prime}$. Comparing the dimensions, we see that $\eta_{3,2}(N)=\operatorname{Im} \eta_{3,2} \cap M_{2}^{\prime}$. This submodule of $M_{2}$ has the same dimension and the same composition factors as $\mathcal{S}_{2}$. Since any submodule of $M_{2}$ with $D_{2}$ as a composition factor must contain $\mathcal{S}_{2}$, we now conclude that $\eta_{3,2}(N)=\mathcal{S}_{2}$.

Lemma 5.7. Let $n \equiv 2(\bmod 4), W=\operatorname{Im} \eta_{2,3} \subseteq M_{3}$, and $Y:=M_{2}^{\prime} \subset M_{2}$. Then:

(i) $\operatorname{soc}^{2}(W) \subseteq N$ and $\operatorname{soc}^{2}(W)$ is uniserial with socle layers $\mathbf{1}, D_{2}$.

(ii) $\operatorname{Ker}\left(\left.\eta_{2,3}\right|_{Y}\right)=\operatorname{soc}(Y)$, and $Y / \operatorname{soc}(Y) \cong \eta_{2,3}(Y)=W$.

(iii) $N \cap W=N \cap \operatorname{soc}^{3}(W)=\operatorname{soc}^{2}(W), M_{3} /\left(N+\operatorname{soc}^{3}(W)\right) \cong Q$.

(iv) We have $M_{1} \cong \operatorname{Im} \eta_{1,3} \subseteq N$, $\operatorname{Im} \eta_{1,3} \cap W=\mathbb{F} \cdot T_{3}$, and the submodule

$$
Q^{\prime}:=\left(\operatorname{Im} \eta_{1,3}+\operatorname{soc}^{2}(W)\right) / \operatorname{soc}^{2}(W) \subseteq N^{\prime}:=N / \operatorname{soc}^{2}(W)
$$
is isomorphic to $Q$. Moreover, $N^{\prime \prime}:=N^{\prime} / Q^{\prime}$ is uniserial with socle layers $D_{3}, D_{2}$.

(v) We have $\operatorname{soc}^{2}(W) \subset \mathcal{S}_{3} \subset N$, the submodule $D^{\prime}:=\mathcal{S}_{3} / \operatorname{soc}^{2}(W) \subset N^{\prime}$ is isomorphic to $D_{3}$. Moreover, $\operatorname{soc}\left(N^{\prime}\right)=D^{\prime} \oplus \operatorname{soc}\left(Q^{\prime}\right) \cong D_{3} \oplus D_{1}$ and $N^{\prime} / D^{\prime} \cong N / \mathcal{S}_{3} \cong$ $\eta_{3,2}(N) \cong \mathcal{S}_{2}$.

Proof. (i) The structure of $W$ is given in Lemma 5.4, which implies that $\operatorname{soc}^{2}(W)$ is uniserial with socle layers $1, D_{2}$. Any nonzero quotient of $\operatorname{soc}^{2}(W)$ has $D_{2}$ as its head. But $M_{3} / N \cong M_{1}$ and $D_{2}$ is not a composition factor of $M_{1}$. So we see that $\operatorname{soc}^{2}(W) \subseteq N$.

(ii) Recall that $M_{2} \cong Y \oplus \mathbf{1}$ and $Y$ is uniserial with socle layers $D_{1}, \mathbf{1}, D_{2}, \mathbf{1}, D_{1}$ by Lemma 5.3. Next, $\eta_{2,3}(Y)$ has codimension $\leq 1$ in $W=\operatorname{Im} \eta_{2,3}$. Inspecting the submodule structures of $W$ and $Y$ given in Lemma 5.4(ii) and Lemma 5.3, we see that $\eta_{2,3}(Y)=W$ and $\operatorname{Ker}\left(\left.\eta_{2,3}\right|_{Y}\right)=\operatorname{soc}(Y)$.

(iii) Note that $\eta_{3,1} \circ \eta_{2,3} \neq 0$, so $W \nsubseteq \operatorname{Ker} \eta_{3,1}=N$. Moreover, $\operatorname{soc}^{3}(W) \nsubseteq N$, since otherwise $W \cap N=\operatorname{soc}^{3}(W)$, and $M_{3} / N \cong M_{1}$ contains $(W+N) / N \cong W / \operatorname{soc}^{3}(W) \cong D_{1}$ 
as a submodule, which is a contradiction. As $W$ is uniserial and $\operatorname{soc}^{2}(W) \subseteq N$, it now follows that $N \cap W=N \cap \operatorname{soc}^{3}(W)=\operatorname{soc}^{2}(W)$. Now, $M_{3} /\left(N+\operatorname{soc}^{3}(W)\right)$ is a quotient of $M_{3} / N \cong M_{1}$ by

$$
\left(N+\operatorname{soc}^{3}(W)\right) / N \cong \operatorname{soc}^{3}(W) /\left(N \cap \operatorname{soc}^{3}(W)\right)=\operatorname{soc}^{3}(W) / \operatorname{soc}^{2}(W) \cong \mathbf{1},
$$

so this quotient must be isomorphic to $Q$.

(iv) We know that $N^{\prime}$ has composition factors $D_{3}, D_{2}, D_{1}$, and $\mathbf{1}$. It is easy to check that $\eta_{3,1} \circ \eta_{1,3}=0$, so $\operatorname{Im} \eta_{1,3} \subseteq N=$ Ker $\eta_{3,1}$. By Lemma 5.5(i), $\operatorname{Im} \eta_{1,3} \cong M_{1}$. Using the submodule structure of $W$ and $\operatorname{Im} \eta_{1,3}$ and the fact that $\operatorname{soc}\left(M_{3}\right)=\mathbb{F} \cdot T_{3}$, we conclude that $\operatorname{Im} \eta_{1,3} \cap W=\mathbb{F} \cdot T_{3}$. Therefore the image $Q^{\prime}$ of $\operatorname{Im} \eta_{1,3}$ in $N^{\prime}$ is isomorphic to $Q$.

Now we know that $N^{\prime \prime}$ has composition factors $D_{3}$ and $D_{2}$. Note that $\mathcal{S}_{3}^{*}$ is a quotient of $M_{3}$, so some submodule $S^{\prime}$ of $\mathcal{S}_{3}^{*}$ is a quotient of $N$. Also, $D_{3}$ is the head of $\mathcal{S}_{3}$ and the socle of $\mathcal{S}_{3}^{*}$. But $D_{3}$ is not a composition factor of $\operatorname{soc}^{2}(W)+\operatorname{Im} \eta_{1,3}$. It follows that $\mathcal{S}_{3}^{*}$ is a quotient of $M_{3} /\left(\operatorname{soc}^{2}(W)+\operatorname{Im} \eta_{1,3}\right)$, whence $S^{\prime}$ is a quotient of $N /\left(\operatorname{soc}^{2}(W)+\operatorname{Im} \eta_{1,3}\right)=$ $N^{\prime \prime}$ and also of $N^{\prime}$. By [J1] the composition factors of $\mathcal{S}_{3}$ (and $\mathcal{S}_{3}^{*}$ ) are $D_{3}, D_{2}, \mathbf{1}$. Among these, only 1 is a composition factor of $M_{1} \cong M_{3} / N$, so $S^{\prime}$ has both $D_{3}$ and $D_{2}$ as composition factors. We have shown that $S^{\prime}$ is a quotient of $N^{\prime \prime}$ which has exactly two composition factors $D_{3}$ and $D_{2}$. It follows that in fact $N^{\prime \prime} \cong S^{\prime}$. In this case, $\operatorname{soc}\left(N^{\prime \prime}\right) \cong D_{3}$ since $\operatorname{soc}\left(\mathcal{S}_{3}^{*}\right) \cong D_{3}$ is simple.

(v) Recall that $\mathcal{S}_{3} \subset M_{3}$ and head $\left(\mathcal{S}_{3}\right) \cong D_{3}$ is not a composition factor of $M_{1} \cong$ $M_{3} / N$. It follows that $\mathcal{S}_{3} \subset N$ and, since $D_{1}$ is not a composition factor of $\mathcal{S}_{3}$, the image $D^{\prime}$ of $\mathcal{S}_{3}$ in $N^{\prime}$ intersects $Q^{\prime}$ trivially. The aforementioned structure of $N^{\prime \prime}$ implies that $\mathcal{S}_{3}$ has no quotient isomorphic to $N^{\prime \prime}$. Therefore, under the natural projection $N^{\prime} \rightarrow N^{\prime \prime}, D^{\prime}$ projects onto a module isomorphic to $D_{3}$, or 0 . The latter cannot happen since $D_{3}$ is not a composition factor of $\operatorname{soc}^{2}(W)+\operatorname{Im} \eta_{1,3}$. So $D^{\prime}$ projects onto a module isomorphic to $D_{3}$. This implies that the composition factors of $D^{\prime}+Q^{\prime}$ are $D_{3}, D_{1}, \mathbf{1}$. Since $D^{\prime} \cap Q^{\prime}=0$ and $Q^{\prime} \cong Q$, it follows that $D^{\prime} \cong D_{3}$. We have shows that that $D_{3} \oplus D_{1} \cong D^{\prime} \oplus \operatorname{soc}\left(Q^{\prime}\right) \subseteq$ $\operatorname{soc}\left(N^{\prime}\right)$. On the other hand, $N^{\prime} / Q^{\prime}=N^{\prime \prime}, \operatorname{soc}\left(N^{\prime \prime}\right) \cong D_{3}$, and $\operatorname{soc}\left(Q^{\prime}\right) \cong D_{1}$. Together these imply that $\operatorname{soc}\left(N^{\prime}\right)$ embeds into $D_{3} \oplus D_{1}$. So $D^{\prime} \oplus \operatorname{soc}\left(Q^{\prime}\right)=\operatorname{soc}\left(N^{\prime}\right)$.

Let $\pi$ denote the natural projection $N \rightarrow N^{\prime}$. Then we have shown that $\operatorname{Ker}\left(\left.\pi\right|_{\mathcal{S}_{3}}\right)$ has two composition factors $D_{2}$ and 1. On the other hand, $\operatorname{Ker} \pi=\operatorname{soc}^{2}(W)$. It follows that $\operatorname{Ker}\left(\left.\pi\right|_{\mathcal{S}_{3}}\right)=\operatorname{soc}^{2}(W)$ so that in fact $D^{\prime}=\mathcal{S}_{3} / \operatorname{soc}^{2}(W)$ and $N^{\prime} / D^{\prime} \cong N / \mathcal{S}_{3}$. By (5.1) we have $\mathcal{S}_{3}=N \cap \operatorname{Ker} \eta_{3,2}$. So $N / \mathcal{S}_{3}=N /\left(\operatorname{Ker} \eta_{3,2} \cap N\right) \cong \eta_{3,2}(N)$ is a submodule of $M_{2}$ with composition factors $D_{1}, \mathbf{1}, D_{2}$, which are precisely the composition factors of $\mathcal{S}_{2}$. Hence, $N^{\prime} / D^{\prime} \cong N / \mathcal{S}_{3}=\eta_{3,2}(N)=\mathcal{S}_{2}$.

\section{MAin ReduCtion theOREM}

The following theorem is the main tool for proving reducibility of various restrictions $\operatorname{Res}_{X}^{S_{n}}$ in the key case $p=2 \mid n$. Note by Theorem 3.2 that the assumption $d_{3}(V)>d_{1}(V)$ is equivalent to the assumption that $V$ is not trivial and not the basic spin module.

Theorem 6.1. Let $p=2 \mid n \geq 6, V$ be an irreducible $\mathbb{F S}_{n}$-module satisfying $d_{3}(V)>$ $d_{1}(V)$, and $X$ be a subgroup of $\mathrm{S}_{n}$. Let $N$ be the $\mathbb{F S}_{n}$-submodule of $M_{3}$ specified in Lemma 5.5(ii). Suppose that for any nonzero $\mathbb{F} S_{n}$-quotient $J$ of $N$ we have $J^{X} \neq 0$ and if $J^{S_{n}} \neq 0$ then $\operatorname{dim} J^{X} \geq 2$. Then the restriction $\operatorname{Res}_{X}^{S_{n}} V$ is reducible. 
Proof. Set $E:=\operatorname{End}_{\mathbb{F}}(V)$ so that $d_{r}(V)=\operatorname{dim}_{H_{0}} \operatorname{Hom}_{n}\left(M_{r}, E\right)$. By Schur's Lemma, $\operatorname{dim} E^{\mathrm{S}_{n}}=\operatorname{End}_{\mathbb{F} \mathrm{S}_{n}}(V)=1$, so the $\mathbb{F} S_{n}$-module $E$ contains a unique submodule $E_{1} \cong \mathbf{1}$. Note that $E^{X}=\operatorname{End}_{\mathbb{F} X}\left(\operatorname{Res}_{X}^{\mathrm{S}_{n}} V\right)$, so it suffices to prove that $\operatorname{dim} E^{X} \geq 2$.

By definition of $N$ in Lemma 5.5(ii), we have an exact sequence

$$
0 \rightarrow N \rightarrow M_{3} \rightarrow M_{1} \rightarrow 0 .
$$

Applying $\operatorname{Hom}_{\mathbb{F} S_{n}}(-, E)$ to this sequence and using the assumption $d_{3}(V)>d_{1}(V)$, we conclude that there is some $f \in \operatorname{Hom}_{\mathbb{F S}}\left(M_{3}, E\right)$ such that $J:=f(N) \neq 0$.

If $J \cap E_{1}=0$, then $E$ contains a submodule isomorphic to $J \oplus E_{1}$, whence $\operatorname{dim} E^{X} \geq 2$ as $J^{X} \neq 0$ by assumption. On the other hand, if $J \cap E_{1} \neq 0$ then $J^{\mathrm{S}_{n}} \neq 0$. In this case $\operatorname{dim} E^{X} \geq \operatorname{dim} J^{X} \geq 2$ by assumption again.

Our main goal now will be to obtain permutation group theoretic conditions on the subgroup $X$ which guarantee that the assumptions of Theorem 6.1 hold.

To bound dimensions of various fixed point subspaces, we will frequently use the following well-known estimates:

Lemma 6.2. Let $X$ be a group and $U \supseteq V$ be $\mathbb{F} X$-modules. Then:

(i) $\operatorname{dim}(U / V)^{X}-\operatorname{dim} H^{1}(X, V) \leq \operatorname{dim} U^{X}-\operatorname{dim} V^{X} \leq \operatorname{dim}(U / V)^{X}$.

(ii) If in addition $X$ acts trivially on $V$ and $\operatorname{Hom}(X, \mathbb{F})=0$, then

$$
\operatorname{dim} U^{X}=\operatorname{dim} V+\operatorname{dim}(U / V)^{X} .
$$

Proof. (i) follows from the exact sequence $0 \rightarrow V^{X} \rightarrow U^{X} \rightarrow(U / V)^{X} \rightarrow H^{1}(X, V)$. (i).

(ii) In this case $H^{1}(X, V)=\operatorname{Hom}(X, \mathbb{F})=0$, whence the statement follows from

Let $X \leq \mathrm{S}_{n}$ be any subgroup and $1 \leq r \leq n / 2$. We set

$$
f_{r}(X):=\operatorname{dim}\left(M_{r}\right)^{X}, \quad f_{0}(X):=0, \quad e_{r}(X):=f_{r}(X)-f_{r-1}(X) .
$$

Note that $f_{r}(X)$ is the number of $X$-orbits on $\Omega_{r}$. As in (5.3) let the $\mathbb{F S}_{n}$-module $Q$ be defined by the short exact sequence

$$
0 \rightarrow \mathbb{F} \cdot T_{1} \rightarrow M_{1} \rightarrow Q \rightarrow 0 .
$$

When $p=2 \mid n$ we also put

$$
h(X):=\operatorname{dim} H^{1}(X, Q) .
$$

For any partition $\lambda \vdash n$, let $\chi^{\lambda}$ denote the irreducible ordinary $\mathrm{S}_{n}$-character labeled by $\lambda$. It is well known (and follows for instance from the Littlewood-Richardson formula [J2, 16.4]) that the $S_{n}$-character afforded by the permutation module $\mathbb{C} \Omega_{r}$ is $\sum_{s=0}^{r} \chi^{(n-s, s)}$. Denote $\alpha:=\chi^{(n-1,1)}$, so that $\alpha+1_{\mathrm{S}_{n}}$ is the permutation character of $\mathrm{S}_{n}$ acting on $\Omega:=\{1,2, \ldots, n\}$. Applying [GT2, Lemma 3.3], we see that

$$
\sum_{s=0}^{r} \chi^{(n-s, s)}= \begin{cases}S^{2}(\alpha) & \text { if } r=2, \\ S^{3}(\alpha)-\wedge^{2}(\alpha)-\alpha & \text { if } r=3,\end{cases}
$$


where $S^{k}$ denotes the $k$ th symmetric power and $\wedge^{k}$ denotes the $k$ th exterior power. If we know the restriction $\alpha_{X}:=\operatorname{Res}_{X}^{\mathrm{S}_{n}} \alpha$ explicitly, we can compute $f_{2}(X)$ and $f_{3}(X)$ by computing the scalar product of $X$-characters as follows:

$$
f_{2}(X)=\left[S^{2}\left(\alpha_{X}\right), 1_{X}\right]_{X}, f_{3}(X)=\left[\left(S^{3}\left(\alpha_{X}\right)-\wedge^{2}\left(\alpha_{X}\right)-\alpha_{X}, 1_{X}\right]_{X} .\right.
$$

Next we record some elementary observations:

Lemma 6.3. Let $X \leq \mathrm{S}_{n}$ be a transitive subgroup. Then:

(i) $f_{2}(X)=1$ if and only if $X$ is 2-homogeneous.

(ii) Suppose $|X|$ is even. Then $f_{2}(X)=1$ if and only if $X$ is 2-transitive.

(iii) $f_{2}(X) \leq 2$ if $X$ is a rank $\leq 3$ subgroup of $\mathrm{S}_{n}$.

Proof. (i) is obvious: $X$ is 2-homogeneous precisely when it acts transitively on $\Omega_{2}$. For (ii), observe that $X$ contains an involution $t$, and so we can find $x, y \in \Omega$ interchanged by $t$. It follows that $X$ is 2-homogeneous precisely when it is 2-transitive.

For (iii), note that if $X$ is a rank 2 subgroup $f_{2}(X)=1$ by (ii). If $X$ is a rank 3 subgroup, then the point stabilizer of $x \in \Omega$ in $X$ has two orbits on $\Omega \backslash\{x\}$, whence $X$ has at most two orbits on $\Omega_{2}$.

Note that since $p=2$ in the following proposition, the condition $X=O^{2}(X)$ is equivalent to the condition $\operatorname{Hom}(X, \mathbb{F})=0$ from Lemma 6.2. In many applications $X$ will be perfect, in which case this assumption of course holds.

Proposition 6.4. Let $p=2 \mid n \geq 6, N$ the $\mathbb{F} S_{n}$-submodule of $M_{3}$ specified in Lemma 5.5(ii), and let $J$ be any nonzero $\mathbb{F S}_{n}$-quotient of $N$. Suppose that $X=O^{2}(X) \leq \mathrm{S}_{n}$ is a subgroup such that

$$
f_{1}(X)=1, e_{3}(X) \geq h(X)+1, \text { and either } f_{2}(X) \geq 3 \text { or } \mathcal{S}_{2}^{X} \neq 0 .
$$

Then $J^{X} \neq 0$. Moreover, if $J^{\mathrm{S}_{n}} \neq 0$, then $\operatorname{dim} J^{X} \geq 2$.

Proof. We write $f_{r}$ for $f_{r}(X), e_{r}$ for $e_{r}(X)$, and $h$ for $h(X)$. Note that $Q^{X}=0$ and $D_{1}^{X}=0$ since $f_{1}=1$ and $X=O^{2}(X)$. Combining this with the structure of $M_{2}$ given in Lemma 5.3 and applying Lemma 6.2, we see that

$$
f_{2} \geq 1+\operatorname{dim} D_{2}^{X}-\operatorname{dim} H^{1}(X, Q)=1+\operatorname{dim} D_{2}^{X}-h .
$$

Note that $D_{2}^{X} \neq 0$. Indeed, if $f_{2} \geq 3$, this follows by considering composition factors of $M_{2}$ described in Lemma 5.3 and using $Q^{X}=D_{1}^{X}=0$. On the other hand, if $\mathcal{S}_{2}^{X} \neq 0$, this follows by considering composition factors of $\mathcal{S}_{2}$ using $D_{1}^{X}=0$.

Case 1: $n \equiv 0(\bmod 4)$. Then $N$ is uniserial by Lemma 5.4 , and we are going to check that $J^{X} \neq 0$ for each of its five non-trivial quotients $J$. This is all we have to do, since 1 is not a composition factor of $N$, and so we never have $J^{S_{n}} \neq 0$.

Note that $\operatorname{soc}(N) \cong D_{2}$, so $N^{X} \supseteq D_{2}^{X} \neq 0$. By assumption, we have $f_{3} \geq f_{2}+$ $h+1$, so (6.4) implies $f_{3} \geq \operatorname{dim} D_{2}^{X}+2$. Since $M_{3}=M_{1} \oplus N$ and $f_{1}=1$, it follows that $(N / \operatorname{soc}(N))^{X} \neq 0$. Then since $D_{1}^{X}=0$, we also get $\left(N / \operatorname{soc}^{2}(N)\right)^{X} \neq 0$. Next, $N / \operatorname{soc}^{3}(N) \cong \mathcal{S}_{2}$ by Lemma 5.6. If $\mathcal{S}_{2}^{X} \neq 0$, we are done. Otherwise, the conditions $f_{2} \geq 3$ and $D_{1}^{X}=0$ imply that $\left(N / \operatorname{soc}^{3}(N)\right)^{X} \neq 0$. Finally, $N / \operatorname{rad}(N) \cong D_{2}$ and we already have $D_{2}^{X} \neq 0$. 
Case 2: $n \equiv 0(\bmod 4)$. We are going to use the notation of Lemma 5.7.

Step 1: we prove that $J^{X} \neq 0$ for any nonzero quotient $J=N / K$ of $N$.

By Lemma 5.7(i), we have the submodule $\operatorname{soc}^{2}(W) \subset N$ which is uniserial with socle layers $\mathbf{1}, D_{2}$. So any nonzero quotient of $\operatorname{soc}^{2}(W)$ either contains $\mathbf{1}$ or is isomorphic to $D_{2}$, hence it contains nonzero $X$-fixed points. In particular, $(N / K)^{X}>0$ if $\operatorname{soc}^{2}(W) \nsubseteq K$, and we may now assume that $\operatorname{soc}^{2}(W) \subseteq K$. In other words, we are reduced to showing that $X$ has nonzero fixed points on every nonzero $\mathbb{F} S_{n}$-quotient of $N^{\prime}=N / \operatorname{soc}^{2}(W)$.

Recall that $M_{2} \cong Y \oplus \mathbf{1}$, see Lemma 5.7. In particular, $\operatorname{dim} Y^{X}=f_{2}-1$, and so $\operatorname{dim}\left(\operatorname{soc}^{4}(Y)\right)^{X}=f_{2}-1$, since $X$ has no fixed points on $U / \operatorname{soc}^{4}(U) \cong D_{1}$. Applying Lemma 6.2(i) to the exact sequence

$$
0 \rightarrow \operatorname{soc}^{2}(Y) \rightarrow \operatorname{soc}^{4}(Y) \rightarrow \operatorname{soc}^{4}(Y) / \operatorname{soc}^{2}(Y) \rightarrow 0
$$

with $\left(\operatorname{soc}^{2}(Y)\right)^{X} \cong Q^{X}=0$, we see that

$$
\operatorname{dim}\left(\operatorname{soc}^{4}(Y) / \operatorname{soc}^{2}(Y)\right)^{X} \leq f_{2}+h-1 .
$$

By Lemma 5.7(ii), we have $\eta_{2,3}(Y)=W \cong Y / \operatorname{soc}(Y)$. So $\operatorname{soc}^{3}(W) \cong \operatorname{soc}^{4}(Y) / \operatorname{soc}(Y)$ is an extension of $\operatorname{soc}^{4}(Y) / \operatorname{soc}^{2}(Y)$ by $\operatorname{soc}^{2}(Y) / \operatorname{soc}(Y) \cong \mathbf{1}$. Together with (6.5) and Lemma 6.2, this implies that

$$
\operatorname{dim}\left(\operatorname{soc}^{3}(W)\right)^{X} \leq f_{2}+h
$$

Since $Q^{X}=0$, Lemma $5.7($ iii) yields

$$
\operatorname{dim}\left(N+\operatorname{soc}^{3}(W)\right)^{X}=f_{3} .
$$

Moreover, by the same lemma, we have

$$
N^{\prime}=N / \operatorname{soc}^{2}(W)=N /\left(N \cap \operatorname{soc}^{3}(W)\right) \cong\left(N+\operatorname{soc}^{3}(W)\right) / \operatorname{soc}^{3}(W) .
$$

Since $f_{3}-f_{2}=e_{3} \geq h+1$ we deduce from (6.6) and (6.7) that $\left(N^{\prime}\right)^{X} \neq 0$.

Now we apply Lemma 5.7(iv). Since $N^{\prime \prime}=N^{\prime} / Q^{\prime}$ and $Q^{\prime X} \cong Q^{X}=0$, we have that $\left(N^{\prime}\right)^{X} \neq 0$ implies $N^{\prime \prime X} \neq 0$. Recalling that $D_{2}^{X} \neq 0$ and head $\left(N^{\prime \prime}\right) \cong D_{2}$, we have now shown that $X$ has nonzero fixed points on every nonzero quotient of $N^{\prime \prime}$.

It remains to consider quotients of $N^{\prime}$ by nonzero submodules $R^{\prime}$ which do not contain $Q^{\prime}$. Since $Q^{\prime}$ has a simple socle and $Q^{\prime} / \operatorname{soc}\left(Q^{\prime}\right) \cong \mathbf{1}$, we only need to consider $R^{\prime}$ such that $R^{\prime} \cap Q^{\prime}=0$. We have shown in Lemma 5.7(v) that $\operatorname{soc}\left(N^{\prime}\right)=D^{\prime} \oplus \operatorname{soc}\left(Q^{\prime}\right)$. Hence the condition $R^{\prime} \cap Q^{\prime}=0$ implies that $R^{\prime} \supseteq D^{\prime}$. So we must show that $X$ has nonzero fixed points on every nonzero quotient of the $\mathbb{F} S_{n}$-module $N^{\prime} / D^{\prime} \cong \mathcal{S}_{2}$, see Lemma 5.7(v) again. By assumption, $\mathcal{S}_{2}^{X} \neq 0$ unless $f_{2} \geq 3$, in which case $D_{1}^{X}=0$ implies $\mathcal{S}_{2}^{X} \neq 0$. The only proper quotients of $\mathcal{S}_{2}$ have socles 1 and $D_{2}$ and we have seen already that $X$ has non-trivial fixed points on these simple modules.

Step 2: we assume that $J=N / K$ contains a trivial $\mathrm{S}_{n}$-submodule $E \cong \mathbf{1}$ and prove that $\operatorname{dim} J^{X} \geq 2$. We again use the notation of Lemma 5.7. Since we have shown that $D_{2}^{X},\left(N^{\prime \prime}\right)^{X} \neq 0$, by Lemma 6.2(ii) it suffices to show that $J$ contains an $\mathbb{F} S_{n^{-}}$-submodule $L$ where $L / E$ is isomorphic to $D_{2}$ or $N^{\prime \prime}$. This is obvious if $K=0$. So we may assume that $K \supseteq \operatorname{soc}(W)=\operatorname{soc}(N) \cong \mathbf{1}$.

If $K \cap \operatorname{soc}^{2}(W)=\operatorname{soc}(W)$, then $J$ contains $\operatorname{soc}^{2}(W) / \operatorname{soc}(W) \cong D_{2}$, and we can take $L \cong D_{2} \oplus E$. Thus we may assume that $K \supseteq \operatorname{soc}^{2}(W)$ and so $J$ can be regarded as 
a quotient $N^{\prime} / K^{\prime}$ of $N^{\prime}=N / \operatorname{soc}^{2}(W)$. Let $P^{\prime}$ be the preimage of $E$ in $N^{\prime}$ so that $E \cong P^{\prime} / K^{\prime}$. Since 1 is not a composition factor of $N^{\prime} / Q^{\prime}$, we see that $Q^{\prime}+K^{\prime} \supseteq P^{\prime}$, whence $Q^{\prime} /\left(Q^{\prime} \cap K^{\prime}\right) \cong\left(Q^{\prime}+K^{\prime}\right) / K^{\prime}$ contains $E=P^{\prime} / K^{\prime}$. But $Q^{\prime}$ has socle layers $D_{1}, \mathbf{1}$, so $Q^{\prime} \cap K^{\prime}=\operatorname{soc}\left(Q^{\prime}\right)$ and $P^{\prime}=Q^{\prime}+K^{\prime}$. Recall that $N^{\prime} / Q^{\prime}=N^{\prime \prime}$ is uniserial with socle layers $D_{3}, D_{2}$. So if $P^{\prime} \neq N^{\prime}$ then $J \cong N^{\prime} / K^{\prime}$ is isomorphic to an extension of the nonzero quotient $N^{\prime} / P^{\prime}$ of $N^{\prime} / Q^{\prime} \cong N^{\prime \prime}$ by $P^{\prime} / K^{\prime}=E$, and we may take $L=J$.

Assume $P^{\prime}=N^{\prime}$, so that $N^{\prime} / K^{\prime} \cong 1$. Since $D^{\prime} \cong D_{3}$ is simple, it follows that $D^{\prime} \subset K^{\prime}$. Now we see that $N^{\prime} / K^{\prime} \cong \mathbf{1}$ is a quotient of $N^{\prime} / D^{\prime}$ which is isomorphic to $\mathcal{S}_{2}$ by Lemma $5.7(\mathrm{v})$, a contradiction $\operatorname{since} \operatorname{head}\left(\mathcal{S}_{2}\right) \cong D_{2}$.

Now we can prove the main result of this section:

Theorem 6.5. Let $p=2 \mid n \geq 6, V$ be an irreducible $\mathbb{F S}_{n}$-module satisfying $d_{3}(V)>$ $d_{1}(V)$, and $X=O^{2}(X) \leq \mathrm{S}_{n}$ be a subgroup such that

$$
f_{1}(X)=1, e_{3}(X) \geq h(X)+1 \text {, and either } f_{2}(X) \geq 3 \text { or } \mathcal{S}_{2}^{X} \neq 0 .
$$

Then the restriction $\operatorname{Res}_{X}^{\mathrm{S}_{n}} V$ is reducible.

Proof. Apply Theorem 6.1 and Proposition 6.4.

Next we show that the condition $\mathcal{S}_{2}^{X} \neq 0$ is a fairly mild condition which always holds for transitive permutation groups of even degree $n$ of rank $\geq 3$ :

Lemma 6.6. Let $2 \mid n \geq 6$ and let $G \leq \mathrm{S}_{n}$ be a transitive permutation group of rank $\geq 3$. Then $\mathcal{S}_{2}^{G} \neq 0$.

Proof. Since $2|n| G \mid$, is even and so $G$ contains an involution $j$. Choose $x_{0} \in \Omega=$ $\{1,2, \ldots, n\}$ not fixed by $j$. By assumption, the stabilizer $G_{x_{0}}$ of $x_{0}$ has an orbit $\Delta_{1} \ni$ $j\left(x_{0}\right)$ on $\Omega \backslash\left\{x_{0}\right\}$, and $\Delta_{2}:=\left(\Omega \backslash\left\{x_{0}\right\}\right) \backslash \Delta_{1} \neq \emptyset$. Let $\Phi_{1}$ denote the $G$-orbit of the 2 -subset $\left\{x_{0}, j\left(x_{0}\right)\right\}$. Since the $G$-orbit of the ordered pair $\left(x_{0}, j\left(x_{0}\right)\right)$ has length $n\left|\Delta_{1}\right|$ and $j$ interchanges $x_{0}$ and $j\left(x_{0}\right)$, we see that $\left|\Phi_{1}\right|=n\left|\Delta_{1}\right| / 2$. Let $\Phi_{2}:=\Omega_{2} \backslash \Phi_{1}$, where $\Omega_{2}$ denotes the set of all 2 -subsets $\{x, y\}$ of $\Omega$. Then

$$
\left|\Phi_{2}\right|=\left|\Omega_{2}\right|-n\left|\Delta_{1}\right| / 2=n\left|\Delta_{2}\right| / 2>0 .
$$

As $\left|\Delta_{1}\right|+\left|\Delta_{2}\right|=n-1 \equiv 1(\bmod 2)$, there is (a unique) $k \in\{1,2\}$ such that $\left|\Delta_{k}\right|$ is even.

Since $\Phi_{k}$ is a non-empty union of $G$-orbits, it suffices to show that the orbit sum $\hat{\Phi}_{k}:=\sum_{\{x, y\} \in \Phi_{k}}\{x, y\}$ belongs to $\mathcal{S}_{2}$. A standard fact about Specht modules following from (5.1) and (5.2) is that

$$
\mathcal{S}_{2}=\left\langle T_{2}\right\rangle^{\perp} \cap \eta_{1,2}\left(M_{1}\right)^{\perp},
$$

where perpendicularity is with respect to the natural inner product $\langle\cdot, \cdot\rangle_{2}$ on $M_{2}$. Now,

$$
\left\langle\hat{\Phi}_{k}, T_{2}\right\rangle_{2}=\left|\Phi_{k}\right|=n\left|\Delta_{k}\right| / 2 \equiv 0(\bmod 2) .
$$

Next, for any $a \in \Omega \backslash\left\{x_{0}\right\},\left\{x_{0}, a\right\} \in \Phi_{1}$ if and only if there is some $g \in G$ sending $\left\{x_{0}, j\left(x_{0}\right)\right\}$ to $\left\{x_{0}, a\right\}$. Replacing $g$ by $g j$ if necessary, we get $g \in G_{x_{0}}$ and $a \in \Delta_{1}$. It follows that $\left\{x_{0}, a\right\} \in \Phi_{k}$ if and only if $a \in \Delta_{k}$, whence

$$
\left\langle\hat{\Phi}_{k}, \eta_{1,2}\left(x_{0}\right)\right\rangle_{2}=\left|\Delta_{k}\right| \equiv 0(\bmod 2) .
$$

By transitivity of $G$ on $\Delta,\left\langle\hat{\Phi}_{k}, \eta_{1,2}(x)\right\rangle_{2}=0$ for all $x \in \Omega$, and the claim follows. 


\section{Special embeddings of $\mathrm{A}_{m}$ into $\mathrm{A}_{n}$}

Let $X \cong \mathrm{A}_{m}$ with $m \geq 5$. In this section, we consider two special kinds of embeddings of $X$ into symmetric groups $\mathrm{S}_{n}$. The first arises from the action of $X$ on $k$-subsets of

$$
\Delta:=\{1,2, \ldots, m\}
$$

with $2 \leq k<m / 2$, giving rise to an embedding of $X$ into $S_{n}$, where $n=\left(\begin{array}{c}m \\ k\end{array}\right)$. The second embedding comes from the action on set partitions of $\Delta$ into $b$ subsets of size $a$, where $m=a b$. This gives rise to an embedding into $\mathrm{S}_{n}$, where $n=(a b) ! /\left((a !)^{b} \cdot b !\right)$.

Lemma 7.1. Suppose that $m \geq 8$. Then for any of the two special embeddings, we have that $n \geq m(m-1) / 2$.

Proof. For the first embedding, observe that the sequence $\left(\begin{array}{c}m \\ k\end{array}\right)$ is increasing for $2 \leq k<$ $m / 2$, whence $\left(\begin{array}{c}m \\ k\end{array}\right) \geq\left(\begin{array}{c}m \\ 2\end{array}\right)$. For the second embedding, denote $N_{a, b}:=(a b) ! /\left((a !)^{b} \cdot b !\right)$ and observe that

$$
\frac{N_{a, b+1}}{N_{a, b}}=\frac{(a b+1)(a b+2) \ldots(a b+a-1)}{(a-1) !} \geq a b+1 \geq 5
$$

as long as $a, b \geq 2$. An induction on $a$ shows that $N_{a, 2}>\left(\begin{array}{c}2 a \\ 2\end{array}\right)$ for $a \geq 4$. Now another induction on $b \geq 2$ using (7.1) shows that $N_{a, b}>\left(\begin{array}{c}a b \\ 2\end{array}\right)$ whenever $a \geq 4$ and $b \geq 2$. Next, an induction on $a$ shows that $N_{a, 3} \geq\left(\begin{array}{c}3 a \\ 2\end{array}\right)$ for $a \geq 2$, with equality only when $a=2$. Now another induction on $b \geq 3$ using (7.1) shows that $N_{a, b}>\left(\begin{array}{c}a b \\ 2\end{array}\right)$ if $a \geq 2, b \geq 3$, and $a b \geq 8$.

Recall that $h(X):=\operatorname{dim} H^{1}(X, Q)$, where the $\mathrm{S}_{n}$-module $Q$ is defined in (5.3).

Lemma 7.2. Let $p=2$. We have:

(i) $\operatorname{dim} H^{2}(X, \mathbb{F})=1$.

(ii) If $n$ is even, then one of the following statements holds:

(a) $\operatorname{dim} H^{1}\left(X, M_{1}\right)=1$ and $h(X) \leq 2$.

(b) $4 \mid m, \mathrm{~A}_{m}$ embeds into $\mathrm{A}_{n}$ via its action on partitions $(m / 2, m / 2)$ of $\Delta$, and $\operatorname{dim} H^{1}\left(X, M_{1}\right)=2, h(X) \leq 3$.

Proof. (i) is a well-known fact about the Schur multiplier of $\mathrm{A}_{m}$ (see e.g. [KlL, Theorem 5.1.4(i)]).

(ii) By Frobenius reciprocity we have that

$$
H^{1}\left(X, M_{1}\right) \cong H^{1}\left(X_{1}, \mathbb{F}\right) \cong \operatorname{Hom}\left(X_{1},(\mathbb{F},+)\right),
$$

where $X_{1}$ is the stabilizer in $X$ of a point on the set $\Omega$, and $(\mathbb{F},+)$ is the additive group of the field $\mathbb{F}$. First we consider the case where $X$ is acting on $k$-sets of $\Delta$. Then

$$
X_{1}=\left(\mathrm{S}_{k} \times \mathrm{S}_{m-k}\right) \cap \mathrm{A}_{m} \cong\left(\mathrm{A}_{k} \times \mathrm{A}_{m-k}\right) \cdot 2 .
$$

Since $p=2$, we have that $\operatorname{Hom}\left(\mathrm{A}_{s},(\mathbb{F},+)\right)=0$ for all $s \geq 1$. Denoting by $C_{2}$ the group of order 2 , it follows that $\operatorname{Hom}\left(X_{1},(\mathbb{F},+)\right) \cong \operatorname{Hom}\left(C_{2},(\mathbb{F},+)\right)$ is one-dimensional.

Next we consider the case $X$ is acting on set partitions of $\Delta$ into $b \geq 2$ subsets of size $a=m / b \geq 2$. Then

$$
X_{1}=\left(\mathrm{S}_{a} \prec \mathrm{S}_{b}\right) \cap \mathrm{A}_{m} .
$$


We may assume that the transposition $(1,2)$ fixes the set partition fixed by $X_{1}$. Then $(1,2)$ belongs to the base subgroup $B=\mathrm{S}_{a}^{b}$, whence $\left[B: B \cap X_{1}\right]=2$ and $X_{1} \cong$ $\left(B \cap X_{1}\right) \cdot \mathrm{S}_{b}$. As mentioned above, $\operatorname{Hom}\left(\mathrm{A}_{a},(\mathbb{F},+)\right)=0$. Hence

$$
\operatorname{Hom}\left(X_{1},(\mathbb{F},+)\right) \cong \operatorname{Hom}\left(X_{1} / \mathrm{A}_{a}^{b},(\mathbb{F},+)\right)=\operatorname{Hom}(Y,(\mathbb{F},+)),
$$

where $Y=X_{1} / \mathrm{A}_{a}^{b} \cong 2^{b-1} \cdot \mathrm{S}_{b}$. If $b \geq 3$, then one can check that $[Y, Y]$ contains the normal subgroup $2^{b-1}$, and so

$$
\operatorname{Hom}(Y,(\mathbb{F},+)) \cong \operatorname{Hom}\left(\mathrm{S}_{b},(\mathbb{F},+)\right) \cong \operatorname{Hom}\left(C_{2},(\mathbb{F},+)\right) \cong \mathbb{F}
$$

as $\operatorname{Hom}\left(\mathrm{A}_{b},(\mathbb{F},+)\right)=0$. Assume that $b=2$, i.e. $X_{1}$ fixes the partition $\Delta=\{1,2, \ldots, a\} \cup$ $\{a+1, \ldots, m\}$. If $a \geq 3$ is odd, then the permutation

$$
g:(1, a+1,2, a+2)(3, a+3) \ldots(a, 2 a)
$$

belongs to $X_{1}$ and $g^{2}=(1,2)(a+1, a+2)$ gives rise to an involution in $Y$. Thus $Y$ is cyclic of order 4 , and so again $\operatorname{Hom}(Y,(\mathbb{F},+)) \cong \mathbb{F}$. If $a$ is even, then $Y$ (of order 4 ) is generated by two involutions $(1,2)(a+1, a+2)$ and $(1, a+1)(2, a+2) \ldots(a, 2 a)$, whence $\operatorname{Hom}(Y,(\mathbb{F},+)) \cong \mathbb{F}^{2}$. This proves the claims on $\operatorname{dim} H^{1}\left(X, M_{1}\right)$ in (ii).

Now the bounds on $h(X)$ in (ii) follow immediately from the portion

$$
0=H^{1}(X, \mathbb{F}) \rightarrow H^{1}\left(X, M_{1}\right) \rightarrow H^{1}(X, Q) \rightarrow H^{2}(X, \mathbb{F})
$$

of the long exact sequence arising from (5.3).

Lemma 7.3. Let $X$ embed into $\mathrm{S}_{n}$ via its action on $k$-subsets of $\Delta$ for $2 \leq k<m / 2$. Then $f_{2}(X)=k$.

Proof. We claim that the orbits of $X$ or of $\mathrm{S}_{m}$ on pairs $\{A, B\}$ of distinct $k$-subsets of $\Delta$ are labeled by $j:=|A \cap B|$ for $0 \leq j \leq k-1$, hence $f_{2}(X)=k$. Indeed, the claim is obvious for $\mathrm{S}_{m}$. Since $A \neq B$, we can find $i \in A \backslash B$ and $j \in B \backslash A$. Now the transposition $(i, j)$ fixes the pair $\{A, B\}$, and so $\mathrm{S}_{m}$ and $\mathrm{A}_{m}$ have the same orbits on pairs $\{A, B\}$.

Next we handle the embedding of $\mathrm{A}_{m}$ into $\mathrm{A}_{n}$ via its action on 2-subsets:

Corollary 7.4. Let $p=2$ and $m \geq 6$ be such that $n:=\left(\begin{array}{c}m \\ 2\end{array}\right)$ is even. Let $X=\mathrm{A}_{m}$ embed into $\mathrm{A}_{n}$ via its action on 2 -subsets of $\{1,2, \ldots, m\}$. Suppose that an irreducible $\mathbb{F S}_{n}$-module $V$ satisfies the condition $d_{3}(V)>d_{1}(V)$. Then $\operatorname{Res}_{X}^{\mathrm{S}_{n}} V$ is reducible.

Proof. By Lemma 7.2(ii), we have that $h(X) \leq 2$. On the other hand, $f_{1}(X)=1$, and $f_{2}(X)=2$ by Lemma 7.3. Also, $f_{3}(X) \geq 5$. Indeed, we can regard the $\mathbb{F S}_{n}$-permutation module $M_{1}$ as having a basis consisting of all 2 -subsets $\{i, j\}$ of $\Delta=\{1,2, \ldots, m\}$. Then the module $M_{3}$ has a basis consisting of unordered triples of distinct pairs, and $\mathrm{S}_{m}$ has 5 orbits on this set represented by the triples

$$
\begin{array}{lll}
\{\{1,2\},\{3,4\},\{5,6\}\}, & \{\{1,2\},\{3,4\},\{4,5\}\}, \quad\{\{1,2\},\{2,4\},\{3,4\}\} \\
\{\{1,2\},\{1,3\},\{1,4\}\}, & \{\{1,2\},\{1,3\},\{2,3\}\} .
\end{array}
$$

In particular, $e_{3}(X) \geq 3 \geq h(X)+1$; furthermore, $\mathcal{S}_{2}^{X} \neq 0$ by Lemma 6.6. Hence we are done by Theorem 6.5 . 
Lemma 7.5. Suppose that $m \geq 11$. Then $e_{2}(X) \geq 2$, unless $\mathrm{A}_{m}$ embeds in $\mathrm{A}_{n}$ via its action on 2 -subsets of $\Delta$, in which case $e_{2}(X)=1$.

Proof. Recall that $f_{1}(X)=1$ for the special embeddings of $X$ into $\mathrm{S}_{n}$ in question. Now for the action of $X$ on $k$-subsets of $\Delta$ the result follows from Lemma 7.3.

Now let $X$ act on partitions $P=\left\{P_{1}, \ldots, P_{b}\right\}$ of $\Delta$ into $b a$-subsets $P_{1}, \ldots, P_{b}$. We will exhibit at least 3 orbits of $X$ on pairs of partitions $\{P, Q\}$. Note that $\Delta$ admits two partitions with no common subset between them. It follows that, for each $j=$ $0,1, \ldots, b-2, \Delta$ admits a pair of partitions $\{P, Q\}$, where $P$ and $Q$ contain exactly $j$ common subsets. Certainly, such pairs with different parameters $j$ belong to different $\mathrm{S}_{m}$-orbits. In particular, we are done if $b \geq 4$.

Suppose $b=3$ and $a \geq 4$. Then we get at least one orbit with the above parameter $j=0$. For $j=1$, we get at least two orbits with representatives $\{P, Q\}$, where $P=$ $\left\{P_{1}, P_{2}, R\right\}, Q=\left\{Q_{1}, Q_{2}, R\right\}$, and $\left|P_{1} \cap Q_{1}\right|=1$, respectively $\left|P_{1} \cap Q_{1}\right|=2$.

Suppose $b=2$ and $a \geq 6$. Then for each $s=1,2,3$ we get at least one orbit with representatives $\{P, Q\}$, where $P=\left\{P_{1}, P_{2}\right\}, Q=\left\{Q_{1}, Q_{2}\right\}$, and $\left|P_{1} \cap Q_{1}\right|=s$.

Remark 7.6. It is easy to check that for the embedding via the action of $X$ on $b a$ subsets, we have $e_{2}(X)=0$ if $(a, b)=(3,2)$, and $e_{2}=1$ if $(a, b)=(2,3),(4,2)$, or $(5,2)$. On the other hand, $e_{2}(X)=3$ if $(a, b)=(3,3)$, as one can compute using (6.3) above and $[\mathrm{GAP}]$.

Lemma 7.7. Suppose $m \geq 6$ and $X=\mathrm{A}_{m}$ embeds into $\mathrm{S}_{n}$ via its action on $k$-subsets of $\Delta$ for $2 \leq k<m / 2$. Then either $e_{3}(X) \geq 4$, or $k=2$ and $e_{3}(X)=3$.

Proof. By Lemma 7.3, we have $f_{2}(X)=k$. So we will try to exhibit at least $(k+4)$ $\mathrm{S}_{m^{-}}$orbits on triples $\{A, B, C\}$ of $k$-subsets. We may assume that

$$
|A \cap B| \geq|A \cap C| \geq|B \cap C|
$$

and call $(|A \cap B|,|A \cap C|,|B \cap C|)$ the mark of the triple $\{A, B, C\}$. Certainly, triples with different marks belong to different $\mathrm{S}_{m}$-orbits.

Recall that $m \geq 2 k+1$. First let $|A \cap B|=k-1$, so we may assume

$$
A=\{1,2, \ldots, k-1, k\}, B=\{1,2, \ldots, k-1, k+1\} .
$$

For $0 \leq j \leq k-1$, by choosing

$$
C=\{1,2, \ldots, j, k+2, k+3, \ldots, 2 k+1-j\}
$$

we get a triple with the mark $(k-1, j, j)$. Similarly, for $1 \leq j \leq k-1$, by choosing

$$
C=\{1,2, \ldots, j-1, k, k+2, k+3, \ldots, 2 k+1-j\}
$$

we get a triple with the mark $(k-1, j, j-1)$.

Next we consider the case $|A \cap B|=k-2$, say

$$
A=\{1,2, \ldots, k-2, k-1, k\}, B=\{1,2, \ldots, k-2, k+1, k+2\} .
$$

For $1 \leq j \leq k-2$, by choosing

$$
C=\{1,2, \ldots, j, k+3, k+4, \ldots, 2 k+2-j\}
$$


we get a triple with the mark $(k-2, j, j)$. Similarly, for $1 \leq j \leq k-2$, by choosing

$$
C=\{1,2, \ldots, j-1, k, k+2, k+3, \ldots, 2 k+2-j\}
$$

we get a triple with the mark $(k-2, j, j-1)$.

We have produced at least $4 k-5$ different marks. So we have $e_{3}(X) \geq 3 k-5 \geq 4$ if $k \geq 3$.

Finally, consider the case $k=2$. Then the triples

$$
\{\{1,2\},\{1,3\},\{2,4\}\},\{\{1,2\},\{1,3\},\{4,5\}\},\{\{1,2\},\{3,4\},\{5,6\}\}
$$

have marks $(1,1,0),(1,0,0)$, and $(0,0,0)$. Furthermore, the triples $\{\{1,2\},\{1,3\},\{1,4\}\}$ and $\{\{1,2\},\{1,3\},\{2,3\}\}$ have the same mark $(1,1,1)$, but different cardinality of $A \cup$ $B \cup C$, so they produce two more $\mathrm{S}_{m}$-orbits.

To estimate $e_{3}(X)$ for the second special embedding of $X$ into $S_{n}$, we need the following observation:

Lemma 7.8. Let $Y$ be any group and $\mathbb{K}$ any field. Suppose that $A_{1}, A_{2}, B_{1}, B_{2}$ are $\mathbb{K} Y$ modules such that there is an injective $f \in \operatorname{Hom}_{\mathbb{K} Y}(A, B)$ with $A=A_{1} \oplus A_{2}, B=B_{1} \oplus B_{2}$, and $f\left(A_{2}\right) \subseteq B_{2}$. Then

$$
\operatorname{dim} B^{Y}-\operatorname{dim} A^{Y} \geq \operatorname{dim} B_{1}^{Y}-\operatorname{dim} A_{1}^{Y} .
$$

Proof. Clearly, $A^{Y}=A_{1}^{Y} \oplus A_{2}^{Y}$ and $B^{Y}=B_{1}^{Y} \oplus B_{2}^{Y}$. Now $f$ embeds $A_{2}^{Y}$ in $B_{2}^{Y}$, whence the claim.

The following statement is also well known and follows for example from the formula for $\operatorname{rank}_{\mathbb{K}} \eta_{2,3}$ given in [Wil]:

Lemma 7.9. Let $p \neq 2,3$ and $n \geq 4$. Then $\eta_{2,3}: M_{2} \rightarrow M_{3}$ is injective.

Now we can prove a reduction lemma to help estimate $e_{3}(X)$ for the second special embedding.

Lemma 7.10. Let $X=\mathrm{A}_{m}$ and $Y:=\mathrm{S}_{m}$ embed into $\mathrm{S}_{n}$ via their actions on partitions of $\Delta=\{1,2, \ldots, m\}$ into $b$ a-subsets, with $a, b \geq 2$. Suppose that $b>s \geq 2$. Set $n^{\prime}:=(s a) ! /\left((a !)^{s} \cdot s !\right)$ and let $Z:=\mathrm{S}_{s a}$ embed in $\mathrm{S}_{n^{\prime}}$ via its action on partitions of $\Delta^{\prime}=\{1,2, \ldots$, sa $\}$ into $s$ a-subsets. Also denote by $N_{r}$ the permutation $\mathrm{S}_{n^{\prime}}$-module corresponding to its action on r-subsets of $\left\{1,2, \ldots, n^{\prime}\right\}$. Then

$$
\operatorname{dim} M_{3}^{X}-\operatorname{dim} M_{2}^{X} \geq \operatorname{dim} N_{3}^{Z}-\operatorname{dim} N_{2}^{Z} .
$$

Proof. 1) Since $M_{r}$ is a permutation module, $\operatorname{dim} M_{r}^{X}$ remains unchanged when we replace $\mathbb{F}$ by any other field. So throughout this proof we may assume $\mathbb{F}=\mathbb{C}$.

With respect to $X$ and $Y$, any pair $\{P, Q\}$ in $\Omega_{2}$ is a pair of two partitions

$$
P=\left\{P_{1}, \ldots, P_{a}\right\}, Q=\left\{Q_{1}, \ldots, Q_{a}\right\} .
$$

Call $\{P, Q\}$ a good pair if at least $b-s$ subsets $P_{i}$ occur among the $Q_{j}$. Next, we call a triple $\{P, Q, R\} \in \Omega_{3}$ with $R=\left\{R_{1}, \ldots, R_{a}\right\}$ a good triple if all three pairs $\{P, Q\}$, $\{P, R\},\{Q, R\}$ are good. We also call $\{P, Q, R\}$ a very good triple if there are at least $b-s$ subsets $P_{i}$ which occur both among the $Q_{j}$ and among the $R_{j}$. Then $Y>X$ acts 
on the following sets: $\Omega_{21}$ of all good pairs, $\Omega_{22}:=\Omega_{2} \backslash \Omega_{21}, \Omega_{31}$ of all good triples, and $\Omega_{32}:=\Omega_{3} \backslash \Omega_{31}$. Thus

$$
A_{1}:=\mathbb{C} \Omega_{21}, A_{2}:=\mathbb{C} \Omega_{22}, B_{1}:=\mathbb{C} \Omega_{31}, B_{2}:=\mathbb{C} \Omega_{32}
$$

are $\mathbb{C} Y$-submodules of $M_{2}=A_{1} \oplus A_{2}$ and $M_{3}=B_{1} \oplus B_{2}$. Note that $Y>X$ also acts on the set $\Omega_{311}$ of very good triples, and so $B_{11}:=\mathbb{C} \Omega_{311}$ is an $X$-submodule of $B_{1}$. Certainly, $B_{11}^{X} \subseteq B_{1}^{X}$.

Since $\operatorname{char}(\mathbb{C})=0, \eta_{2,3}$ is injective by Lemma 7.9. Next, if $\{P, Q\} \in \Omega_{22}$, then

$$
\eta_{2,3}(\{P, Q\})=\sum_{R \neq P, Q}\{P, Q, R\}
$$

where all occuring triples $\{P, Q, R\}$ are not good (since $\{P, Q\}$ is not good). Thus $\eta_{2,3}(\{P, Q\}) \in B_{2}$. We have shown that $\eta_{2,3}\left(A_{2}\right) \subseteq B_{2}$. Applying Lemma 7.8 to the homomorphism $f=\eta_{2,3}$, we see that

$$
\operatorname{dim} M_{3}^{X}-\operatorname{dim} M_{2}^{X} \geq \operatorname{dim} B_{1}^{X}-\operatorname{dim} A_{1}^{X} \geq \operatorname{dim} B_{11}^{X}-\operatorname{dim} A_{1}^{X} .
$$

Recall that $b>s$. Hence, for any good pair $\{P, Q\}, P$ and $Q$ have at least one common $a$-subset $P_{1}$, and since $a \geq 2$, some transposition $(i, j) \in Y \backslash X$ fixes both $P$ and $Q$. It follows that $X$ and $Y$ have the same orbits on good pairs. Similarly, $X$ and $Y$ have the same orbits on very good triples. Thus

$$
\operatorname{dim} B_{11}^{X}-\operatorname{dim} A_{1}^{X}=\operatorname{dim} B_{11}^{Y}-\operatorname{dim} A_{1}^{Y} .
$$

2) It remains to prove that

$$
\operatorname{dim} B_{11}^{Y}-\operatorname{dim} A_{1}^{Y}=\operatorname{dim} N_{3}^{Z}-\operatorname{dim} N_{2}^{Z} .
$$

To do so, we will count the $X$-orbits on good pairs and very good triples.

Let $r \in\{2,3\}$. Suppose the good pairs (if $r=2$ ), respectively the very good triples (if $r=3),\left\{P^{1}, \ldots, P^{r}\right\}$ and $\left\{Q^{1}, \ldots, Q^{r}\right\}$ belong to the same $X$-orbit. Then, without loss we may assume that

$$
P^{i}=\left\{R_{1}, \ldots, R_{b-s}, P_{1}^{i}, \ldots, P_{s}^{i}\right\}, Q^{i}=\left\{R_{1}, \ldots, R_{b-s}, Q_{1}^{i}, \ldots, Q_{s}^{i}\right\}
$$

for $1 \leq i \leq r$. Denote

$$
\bar{P}^{i}:=\left\{P_{1}^{i}, \ldots, P_{s}^{i}\right\}, \bar{Q}^{i}:=\left\{Q_{1}^{i}, \ldots, Q_{s}^{i}\right\}
$$

for $1 \leq i \leq r$.

By assumption, there is some $\sigma \in \mathrm{S}_{m}$ sending $\left\{P^{1}, \ldots, P^{r}\right\}$ to $\left\{Q^{1}, \ldots, Q^{r}\right\}$. Let $t$ be the number of common $a$-subsets that occur in all $P^{1}, \ldots, P^{r}$. Then $t$ is also the number of common $a$-subsets that occur in all $Q^{1}, \ldots, Q^{r}$, and $t \geq b-s$.

Consider the case $t=b-s$. Then the $t$ common $a$-subsets among all $P^{i}$ are precisely $R_{1}, \ldots, R_{b-s}$, and similarly, the $t$ common $a$-subsets among all $Q^{i}$ are precisely $R_{1}, \ldots, R_{b-s}$. It follows that $\sigma$ acts on the set $\left\{R_{1}, \ldots, R_{b-s}\right\}$, and preserves the set

$$
\cup_{j=1}^{s} P_{j}^{i}=\cup_{j=1}^{s} Q_{j}^{i}
$$

which can be identified with $\Delta^{\prime}$. Now we can write $\sigma=\mu \tau$, where

$$
\mu \in \mathrm{S}_{m-s a}=\operatorname{Sym}\left(\cup_{j=1}^{b-s} R_{j}\right)
$$


acts on the set $\left\{R_{1}, \ldots, R_{b-s}\right\}$, and $\tau \in Z=\operatorname{Sym}\left(\Delta^{\prime}\right)$ sends $\left\{\bar{P}^{1}, \ldots, \bar{P}^{r}\right\}$ to $\left\{\bar{Q}^{1}, \ldots, \bar{Q}^{r}\right\}$. Thus, the two pairs, respectively triples, $\left\{\bar{P}^{1}, \ldots, \bar{P}^{r}\right\}$ and $\left\{\bar{Q}^{1}, \ldots, \bar{Q}^{r}\right\}$ of partitions of $\Delta^{\prime}$ belong to the same $Z$-orbit.

Next assume that $t>b-s$ and set $v=t+s-b$. Then the $t$ common $a$-subsets among all $P^{i}$ are precisely $R_{1}, \ldots, R_{b-s}, S_{1}, \ldots, S_{v}$, and similarly, the $t$ common $a$-subsets among all $Q^{i}$ are precisely $R_{1}, \ldots, R_{b-s}, T_{1}, \ldots, T_{v}$, for some $a$-subsets $S_{j}$ and $T_{j}$ of $\Delta$. It follows that $\sigma$ sends $\left\{R_{1}, \ldots, R_{b-s}, S_{1}, \ldots, S_{v}\right\}$ to $\left\{R_{1}, \ldots, R_{b-s}, T_{1}, \ldots, T_{v}\right\}$, and $\Sigma$ to $\Theta$, where

$$
\Sigma=\Delta \backslash\left(\bigcup_{j=1}^{b-s} R_{j} \cup \bigcup_{j=1}^{v} S_{j}\right), \Theta=\Delta \backslash\left(\bigcup_{j=1}^{b-s} R_{j} \cup \bigcup_{j=1}^{v} T_{j}\right) .
$$

Set $R_{j}^{\prime}:=R_{j}$ for $1 \leq j \leq b-s$. Also set $R_{b-s+j}:=S_{j}$ and $R_{b-s+j}^{\prime}:=T_{j}$ for $1 \leq j \leq v$. Then there is a permutation $\pi \in \mathrm{S}_{t}$ such that $\sigma\left(R_{j}\right)=R_{\pi(j)}^{\prime}$. Now we can find $\gamma \in \mathrm{S}_{m}=$ $\operatorname{Sym}(\Delta)$ such that

$$
\gamma_{\Sigma}=1_{\Sigma}, \gamma\left(R_{j}\right)=R_{\pi^{-1}(j)} .
$$

Clearly, $\sigma \gamma$ sends $R_{j}$ to $R_{j}$ for $1 \leq j \leq b-s$ (and $S_{j}$ to $T_{j}$ ), and sends $\left\{P^{1}, \ldots, P^{r}\right\}$ to $\left\{Q^{1}, \ldots, Q^{r}\right\}$. Now we can repeat the argument of the preceding case $t=b-s$ to show that the two pairs, respectively triples, $\left\{\bar{P}^{1}, \ldots, \bar{P}^{r}\right\}$ and $\left\{\bar{Q}^{1}, \ldots, \bar{Q}^{r}\right\}$ of partitions of $\Delta^{\prime}$ belong to the same $Z$-orbit.

Conversely, it is obvious that if $\left\{\bar{P}^{1}, \ldots, \bar{P}^{r}\right\}$ and $\left\{\bar{Q}^{1}, \ldots, \bar{Q}^{r}\right\}$ belong to the same $Z$-orbit, then $\left\{P^{1}, \ldots, P^{r}\right\}$ and $\left\{Q^{1}, \ldots, Q^{r}\right\}$, defined as in (7.3), belong to the same $Y$ orbit. We have therefore proved that $\operatorname{dim} B_{11}^{Y}=\operatorname{dim} N_{3}^{Z}$ and $\operatorname{dim} A_{1}^{Y}=\operatorname{dim} N_{2}^{Z}$, whence (7.2) holds.

Theorem 7.11. Let $p=2$ and $n$ be even. Suppose that $n>m \geq 11$ and $X \cong \mathrm{A}_{m}$ embeds into $\mathrm{A}_{n}$ via its actions on subsets or partitions of $\{1,2, \ldots, m\}$. Then $f_{2}(X) \geq 3$ and $e_{3}(X) \geq h(X)+2$, unless $\mathrm{A}_{m}$ embeds into $\mathrm{A}_{n}$ via its action on 2 -subsets of $\{1,2, \ldots, m\}$.

Proof. 1) The inequality $e_{2}(X) \geq 2$, and hence $f_{2}(X) \geq 3$, is proved in Lemma 7.5.

If $\mathrm{A}_{m}$ embeds into $\mathrm{A}_{n}$ via its action of $k$-subsets of $\Delta$ with $2<k<m / 2$, then $h(X) \leq 2$ by Lemma 7.2, and $e_{3}(X) \geq 4$ by Lemma 7.7. Thus $e_{3}(X) \geq h(X)+2$ as stated.

2) From now on, we assume that $A_{m}$ embeds in $A_{n}$ via its action on partitions of $\Delta$ into $b a$-subsets, $a, b \geq 2$. By Lemma 7.2, either $h(X) \leq 2$, or $h(X)=3$ and $b=2 \mid(m / 2)$.

Here we consider the case $a \geq 5$ and show that $e_{3}(X) \geq 5 \geq h(X)+2$ in this case. Applying Lemma 7.10 with $s=2$, we are reduced to prove that $\operatorname{dim} M_{3}^{Y}-\operatorname{dim} M_{2}^{Y} \geq 5$ for $b=2$ and $Y=\mathrm{S}_{m}$. In this base case $b=2$, each partition is a pair

$$
[A]:=\{A, \Delta \backslash A\}
$$

with $|A|=a=|\Delta| / 2$. Hence the $Y$-orbits on pairs of partitions $\{[A],[B]\}$ are labeled by $1 \leq j \leq t:=\lfloor a / 2\rfloor$, where $|A \cap B|=a-j$. In particular, $\operatorname{dim} M_{2}^{Y}=t$.

So we need to produce at least $(t+5) Y$-orbits on triples of partitions $\{[A],[B],[C]\} \in$ $\Omega_{3}$. As in the proof of Lemma 7.7, we will label $A, B, C$ so that

$$
|A \cap B| \geq|A \cap C| \geq|B \cap C| \geq a / 2
$$

and call $(|A \cap B|,|A \cap C|,|B \cap C|)$ the mark of the triple $\{A, B, C\}$. Certainly, triples with different marks belong to different $\mathrm{S}_{m}$-orbits. 
First let $|A \cap B|=a-1$, so we may assume

$$
A=\{1,2, \ldots, a-1, a\}, B=\{1,2, \ldots, a-1, a+1\} .
$$

For $1 \leq j \leq t$, by choosing

$$
C=\{1,2, \ldots, a-j, a+2, a+3, \ldots, a+j+1\}
$$

we get a triple with the mark $(a-1, a-j, a-j)$. In fact, for $j=1$, we have two choices for $C$ :

$$
\{1,2, \ldots, a-1, a+2\} \text {, and }\{1,2, \ldots, a-2, a, a+1\}
$$

which lead to two different $S_{m}$-orbits with mark $(a-1, a-1, a-1)$ for any $a \geq 4$ (since $|A \cap B \cap C|=a-1$ for the first choice and $|A \cap B \cap C|=a-2$ for the second choice). Similarly, for $1 \leq j \leq t-1$, by choosing

$$
C=\{1,2, \ldots, a-j-1, a, a+2, a+3, \ldots, a+j+1\}
$$

we get a triple with the mark $(a-1, a-j, a-j-1)$.

Suppose in addition that $a \geq 6$. Then we choose

$$
A=\{1,2, \ldots, a-2, a-1, a\}, B=\{1,2, \ldots, a-2, a+1, a+2\} .
$$

Taking

$$
C=\{1,2, \ldots, a-2, a+3, a+4\} \text { or }\{1,2, \ldots, a-3, a+3, a+4, a+5\}
$$

we get triples with the mark $(a-2, a-2, a-2)$ and $(a-2, a-3, a-3)$. We have produced at least $(t+1)+(t-1)+2 \geq t+5$ orbits on triples, as desired.

Next assume that $a=5$, and so $t=2$. We have already produced 4 orbits with marks $(4,4,4)$ (two orbits), $(4,4,3)$, and $(4,3,3)$. We can also exhibits 3 more orbits with

$$
\{A, B, C\}=\{12345,12346,12578\},\{12345,12367,12389\},\{12345,12367,12589\}
$$

and mark $(4,3,2),(3,3,3)$, and $(3,3,2)$, respectively. (Note that the last mark deviates from the convention (7.4), but it does not cause any problem since $a=5$, as one can check.)

For the next part of the proof, we also consider the case $a=4$, and so $s=2$. We have already produced 4 orbits with marks $(3,3,3)$ (two orbits), $(3,3,2)$, and $(3,2,2)$. Next, the triples with

$$
\{P, Q, R\}=\{1234,1256,3456\} \text { and }\{1234,1256,1357\}
$$

have the same mark $(2,2,2)$, but belong to different orbits. (Indeed, the former satisfies the identity $R=P+Q:=(P \backslash Q) \cup(Q \backslash P)$, but for the latter no member of $[R]$ can be the sum of a member of $[P]$ with a member of $[Q]$.) Thus we get at least 6 orbits on triples of partitions. (One can show by [GAP] that the number of orbits on triples is indeed 6 for both $\mathrm{A}_{m}$ and $\mathrm{S}_{m}$.)

3 ) It remains to consider the case $2 \leq a \leq 4$. Since $m \geq 11$, we must have that $b \geq 3$, and so $h \leq 2$ by Lemma 7.2. For $a=2,3$, or 4 , we set $s=5,3$, or 2 , respectively. Applying Lemma 7.10, we are reduced to prove that $e^{\prime}:=\operatorname{dim} M_{3}^{Y}-\operatorname{dim} M_{2}^{Y} \geq 4$ for $b=s$ and $Y=\mathrm{S}_{s a}$. This has been done in 2) for $(a, s)=(4,2)$. Using (6.3) and [GAP], one can check that $e^{\prime}=35$ for $(a, s)=(3,3)$. Finally, assume that $(a, s)=(2,5)$. Using 
(6.3) and [GAP], we see that the number of $A_{10}$-orbits on 2-subsets, respectively on 3subsets of $\Omega=\{1,2, \ldots, 945\}$ is 6 , respectively 139 . Since $\mathrm{A}_{10}$ has index 2 in $Y=\mathrm{S}_{10}$, it follows that $\operatorname{dim} M_{2}^{Y} \leq 6$ and $\operatorname{dim} M_{3}^{Y} \geq 139 / 2$, whence $e^{\prime} \geq 64$.

Now we can prove the main result concerning the special embeddings of $A_{m}$ into $A_{n}$ :

Theorem 7.12. Let $X=\mathrm{A}_{m}$ be embedded in $\mathrm{A}_{n}$ via its actions on partitions or on $k$-subsets of $\{1,2, \ldots, m\}$ with $2 \leq k<m / 2$, and $11 \leq m<n$. Let $p=2$ and let $V$ be any $\mathbb{F} \mathrm{A}_{n}$-module of dimension greater than 1 . Then $\operatorname{Res}_{X}^{\mathrm{A}_{n}} V$ is reducible.

Proof. Assume for a contradiction that $V$ is irreducible over $X$. By Lemma 7.1, $n \geq$ $m(m-1) / 2$. Since $m \geq 11$, we have

$$
|X|=\frac{m !}{2}<2^{\frac{m(m-1)-12}{4}} \leq\left(2^{\frac{n-6}{4}}\right)^{2} .
$$

The irreducibility of $V$ on $X$ forces that $\operatorname{dim} V<\sqrt{|X|}<2^{(n-6) / 4}$. This bound implies by Proposition 4.1 that $V$ extends to $\mathrm{S}_{n}$. Thus, without loss we may assume that $V$ is an irreducible $\mathbb{F S}_{n}$-module. Also, since $2^{(n-6) / 4}<2^{\lfloor(n-2) / 2\rfloor}, V$ cannot be a basic spin module.

Now if $n$ is odd, then the $X$-module $V$ is reducible by [KS1, Theorem 3.10]. Hence $2 \mid n$, and $V$ satisfies the conclusion (i) of Theorem 3.2. By Corollary 7.4, $X$ does not embed into $\mathrm{A}_{n}$ via its action on 2 -subsets of $\{1,2, \ldots, m\}$. Hence, by Theorem $7.11, f_{2}(X) \geq 3$ and $e_{3}(X) \geq h(X)+2$. Clearly, $X$ is a perfect subgroup and $f_{1}(X)=1$. Therefore, the $X$-module $V$ is reducible by Theorem 6.5 , a contradiction.

\section{General embeddings of $\mathrm{A}_{m}$ into $\mathrm{A}_{n}$}

Throughout this section, $X \cong \mathrm{A}_{m}$ is a subgroup of $\mathrm{A}_{n}$, with $n>m \geq 5$. Recall the notation $\Omega=\{1,2, \ldots, n\}$ and $\Delta=\{1,2, \ldots, m\}$.

First, we deal with the small cases $5 \leq m \leq 10$ :

Lemma 8.1. Let $X \cong \mathrm{A}_{m}$ be a transitive subgroup of $\mathrm{A}_{n}$ with $n>m$ and $5 \leq m \leq 10$, $V$ be an $\mathbb{F A}_{n}$-module of dimension $>1$ such that $\operatorname{Res}_{X}^{\mathrm{A}_{n}} V$ is irreducible. Then $(m, n)=$ $(5,6),(6,10),(7,15)$, or $(8,15)$.

Proof. 1) First we consider the case where $V$ is not the heart of the natural permutation module of $Y:=\mathrm{A}_{n}$. Suppose for instance that $m=10$. The assumptions that $X$ is a transitive subgroup of $Y$ and $n>m$ imply by [Atl] that $n \geq 45$. This in turn implies by [GT1, Lemma 6.1] that $\operatorname{dim} V \geq\left(n^{2}-5 n+2\right) / 2=901>567 \geq b_{\mathbb{F}}(X)$, where $b_{\mathbb{F}}(X)$ denotes the largest degree of irreducible $\mathbb{F} X$-representations. It follows that $\operatorname{Res}_{X}^{\mathrm{A}_{n}} V$ is reducible. The same arguments apply to the case $m=9$, where we have $n \geq 36$ and $b_{\mathbb{F}}(X) \leq 216$.

Suppose now that $m=8$. According to [Atl] and [JLPW], $n=15$ or $n \geq 28$; furthermore, $b_{\mathbb{F}}(X) \leq 70$. As above, $\operatorname{dim} V>b_{\mathbb{F}}(X)$ if $n \geq 28$. It follows that $n=15$. The same arguments apply to the case $5 \leq m \leq 7$. (In fact, one can show that we must have either $(m, n)=(5,6)$ or $(m, n, p, \operatorname{dim} V)=(8,15,2,64)$.)

2) Now we may assume that $V$ is the heart of the natural permutation module $\mathbb{F} \Omega$ of $Y$. By the assumption, $X$ acts transitively on $\Omega$. If this action is not doubly transitive, 
then the restriction of the permutation character of $\mathbb{C} \Omega$ is $1_{X}+\alpha+\beta$ where $\alpha, \beta$ are (not necessarily irreducible) $X$-characters of degree $>1$. Since the Brauer character of $\mathbb{F} \Omega$ is $\varphi+e \cdot 1_{Y}$ with $e \in\{1,2\}$ and $\varphi \in \operatorname{IBr}_{p}(Y)$, it follows that $V$ is reducible over $X$. Thus $X$ acts doubly transitively on $\Omega$. Now we can read off the possible value of $n$ using [Atl].

Remark 8.2. The special cases $(m, n)$ listed in the statement of Lemma 8.1 are indeed exceptional. For these pairs, we can embed $A_{m}$ into $A_{n}$ so that $A_{m}$ acts doubly transitively on $\Omega$. Take $V$ to be the heart of the permutation module $\mathbb{C} \Omega$. It is well known (and follows for instance from the Mackey's formula) that any doubly transitive group is irreducible on $V$. This is also almost always true for $p>0-$ see [Mo].

We will need the following group-theoretic result:

Lemma 8.3. Let $X \cong \mathrm{A}_{m}$ be a transitive subgroup of $\mathrm{A}_{n}$ with $n>m \geq 11$. Then $n \geq m(m-1) / 2$ and $X$ cannot act doubly transitively on $\Omega$. If $X$ is imprimitive, then $n \geq m(m-1)$. If $X$ is primitive, then one of the following statements holds:

(i) $X$ acts on $\Omega$ via its action on $k$-subsets of $\Delta$ with $2 \leq k<m / 2$ or on partitions of $\Delta$, and $n \geq m(m-1) / 2$.

(ii) $n>|X|^{3 / 10}>m(m-1)$.

Proof. From the classification of doubly transitive permutation representations of $\mathrm{A}_{m}$ [Ma] and the assumption $m \geq 11$, it follows that $\mathrm{A}_{m}$ has no doubly transitive permutation representation of degree $n>m$. Next, consider the transitive action of $X$ on $\Omega$ and let $X_{1}$ be a point stabilizer of this action. Then $\left[X: X_{1}\right]=n>m$. We can find a maximal subgroup $M$ of $X$ containing $X_{1}$.

Now we consider the action of $M$ on $\Delta$. If this action is intransitive, then by maximality of $M, M$ is the stabilizer in $X$ of a $k$-subset of $\Delta$ for some $1 \leq k<m / 2$. On the other hand, if this action is transitive but imprimitive, then $M$ is the stabilizer in $X$ of a partition of $\Delta$ into $b a$-subsets, with $a, b \geq 2$ and $a b=m$. By Lemma 7.1, in either case we have $n \geq[X: M] \geq m(m-1) / 2$ (whence $\left[X: X_{1}\right] \geq m(m-1)$ if $X$ is imprimitive), unless $M$ is intransitive and $k=1$. In this exceptional case, $M=\mathrm{A}_{m-1}$ and $\left[X: X_{1}\right]=n>m=[X: M]$. Thus $X_{1}$ is a proper subgroup of $M$, and so, as is well known (see e.g. [KlL, p. 175]), $\left[M: X_{1}\right] \geq m-1$ and $n \geq m(m-1)$.

Suppose now that $M$ acts primitively on $\Delta$. By Bochert's Theorem 14.2 of [Wie], $\left[\mathrm{S}_{m}: M\right] \geq\lfloor(m+1) / 2\rfloor$ !. Since $m \geq 11$, we have $\frac{\lfloor(m+1) / 2\rfloor !}{2}>\left(\frac{m !}{2}\right)^{3 / 10}$. Therefore $n \geq[X: M]>|X|^{3 / 10}>m(m-1)$ as $m \geq 11$.

We have proved the bound $n \geq m(m-1) / 2$ and also that $n \geq m(m-1)$ if $X$ is imprimitive. Assume now that $X$ is primitive. Then $X_{1}=M$, and the above analysis yields the final claim of the lemma.

Theorem 8.4. Let $X \cong \mathrm{A}_{m}$ be a primitive subgroup of $\mathrm{A}_{n}$ with $n>m \geq 9$. Let $p=2$ and let $V$ be an $\mathbb{F} \mathrm{A}_{n}$-module of dimension $>1$. Then the restriction $\operatorname{Res}_{X}^{\mathrm{A}_{n}} V$ is reducible.

Proof. By Lemma 8.1, we may assume that $m \geq 11$. Assume for a contradiction that $V$ is irreducible over $X$. We apply Lemma 8.3. In the case (i) of Lemma 8.3 we are done by Theorem 7.12 . 
Now suppose that the case (ii) of Lemma 8.3 holds. Then $|X|<n^{10 / 3}$ and $n \geq 155$ as $m \geq 11$. Since $V$ is irreducible over $X$, we must have that

$$
\operatorname{dim} V<n^{5 / 3}<\frac{1}{2}(n-1)(n-2) .
$$

In particular, $\operatorname{dim} V<2^{(n-6) / 4}$ (as $n \geq 155$ ), whence $V$ extends to $\mathrm{S}_{n}$ by Proposition 4.1. We denote the (unique up to isomorphism) extension of $V$ to $\mathrm{S}_{n}$ by the same letter $V$.

By Lemma 8.3, the action of $X=\mathrm{A}_{m}$ on $\Omega$ is not doubly transitive. Hence, if $n$ is odd then, we are done by appealing to [KS1, Theorem 3.10]. Assume that $2 \mid n \geq 155$. Then the condition $1<\operatorname{dim} V<(n-1)(n-2) / 2$ for the irreducible $\mathbb{F} S_{n}$-module $V$ implies by [J3, Theorem 7] that $V \cong D^{(n-k, k)}$ with $k=1$ or 2 . As before, let $1+\alpha$ denote the (complex) permutation character of $\mathrm{S}_{n}$ on $\Omega$.

Suppose $k=1$. Then $V=D^{(n-1,1)}$ is irreducible over $X$. Denoting by $\alpha^{0}$ the restriction of $\alpha$ to $2^{\prime}$-elements in $S_{n}$, we see that the Brauer character of $V$ is $\alpha^{0}-1$. Furthermore, $X$ is perfect and $\left[\operatorname{Res}_{X}^{\mathrm{S}_{n}} \alpha, 1_{X}\right]_{X}=0$ by transitivity of $X$ on $\Omega$. Hence the irreducibility of $V$ over $X$ forces that $\alpha$ is also irreducible over $X$. In other words, $X$ acts doubly transitively on $\Omega$, a contradiction.

Assume now that $k=2$. Then $\wedge^{2}\left(D^{(n-1,1)}\right)$ has a composition series with composition factors $V$ (once), and $\mathbf{1}$ (once if $4 \mid n$ and twice if $n \equiv 2(\bmod 4))$. The same is true for $\wedge^{2}\left(D^{(n-1,1)}\right)$ considered as an $X$-module. Suppose in addition that $D^{(n-1,1)}$ is reducible over $X$. Then we can write the Brauer character of the $\mathbb{F} X$-module $\operatorname{Res}_{X}^{\mathrm{S}_{n}} D^{(n-1,1)}$ as $\beta+\gamma$, where $\beta$ and $\gamma$ are Brauer characters of $X, \beta(1) \geq 1$ and $\gamma(1) \geq 3$. Note that

$$
\wedge^{2}(\beta+\gamma)=\wedge^{2}(\beta)+\wedge^{2}(\gamma)+\beta \gamma
$$

It follows that the Brauer character of the $X$-module $\wedge^{2}\left(D^{(n-1,1)}\right)$ is the sum of three Brauer characters, with at least two of degree $\geq 3$. This contradicts the aforementioned composition structure of the $X$-module $\wedge^{2}\left(D^{(n-1,1)}\right)$. Thus $D^{(n-1,1)}$ is irreducible over $X$. But then, arguing as in the previous paragraph, we again arrive at the contradiction that $X$ acts doubly transitively on $\Omega$.

The case $p \neq 2$ can be done using results of [KS1], [KS2] and Proposition 4.3:

Proposition 8.5. Let $X \cong \mathrm{A}_{m}$ be a transitive subgroup of $\mathrm{A}_{n}$ with $n>m \geq 9$. Let $p \neq 2$ and $V$ be an $\mathbb{F} \mathrm{A}_{n}$-module of dimension $>1$. Then $\operatorname{Res}_{X}^{\mathrm{A}_{n}} V$ is reducible.

Proof. By Lemma 8.1, we may assume that $m \geq 11$. Assume for a contradiction that $\operatorname{Res}_{X}^{A_{n}} V$ is irreducible. By Lemma 8.3, $X$ is not doubly transitive, and $n \geq m(m-1) / 2$. If $V$ is extendible to $S_{n}$, we can apply [KS1, Main Theorem] to get a contradiction. Suppose $V$ is not extendible to $S_{n}$. If $p \neq 3$, we again arrive at a contradiction by using [KS2, Main Theorem]. So we may assume that $p=3$. By Proposition 4.3(ii) we have that

$$
\operatorname{dim} V \geq 2^{\frac{n-8}{4}} \geq 2^{\frac{m^{2}-m-16}{8}}=: c_{m} .
$$

Now, if $m \geq 12$ then $c_{m}>\sqrt{m ! / 2}=\sqrt{|X|}$. If $m=11$, then $c_{m}>3444$ whereas the largest degree of complex irreducible representations of $X=\mathrm{A}_{11}$ is 2310 . In either case, $X$ cannot be irreducible on $V$. 
Now Theorem 1.2 follows immediately from Theorem 8.4 and Proposition 8.5.

Note that for $p \neq 2$, Proposition 8.5 shows that any proper transitive subgroup $X \cong$ $\mathrm{A}_{m}$ of $\mathrm{A}_{n}$ acts reducibly on all non-trivial modules over $\mathbb{F} \mathrm{A}_{n}$ (provided $m \geq 9$ ). For $p=2$, if $X$ is primitive, the same result holds by Theorem 8.4. Now we handle imprimitive embeddings of $\mathrm{A}_{m}$ into $\mathrm{A}_{n}$ for $p=2$. Here our result is a little weaker:

Proposition 8.6. Let $X \cong \mathrm{A}_{m}$ be a (transitive) imprimitive subgroup of $\mathrm{A}_{n}$ with $n>$ $m \geq 9$. Let $p=2$ and let $V$ be an irreducible $\mathbb{F} \mathrm{A}_{n}$-module of dimension $>1$. Then the $X$-module $\operatorname{Res}_{X}^{\mathrm{A}_{n}} V$ cannot be primitive irreducible.

Proof. By Lemma 8.1, we may assume that $m \geq 11$. Assume for a contradiction $V$ is irreducible and primitive over $X$. Consider the action of $X$ as a subgroup of $\mathrm{A}_{n}$ on $\Omega$ and let $X_{1}$ be a point stabilizer. Since $X$ is imprimitive, there is a maximal subgroup $M>X_{1}$ of $X$. Now $b:=[X: M] \geq m \geq 11$, and we may assume that $X<Y:=\left(\mathrm{S}_{a}<\mathrm{S}_{b}\right) \cap \mathrm{A}_{n}$ for $a:=n / b>1$. By Lemma 8.3, $n \geq m(m-1)$. If $a=2$ or 4 , then $O_{2}(Y)>1$, and so $V$ cannot be irreducible over $Y>X$, a contradiction. So we have $a \geq 3$ and $a \neq 4$.

Let $\lambda=\left(\lambda_{1}, \lambda_{2}, \ldots\right)$ be a 2-regular partition of $n$ such that $V$ is a simple submodule of $\operatorname{Res}_{\mathrm{A}_{n}}^{\mathrm{S}_{n}} D^{\lambda}$. Observe that $m !<(m / 2)^{m}$ for $m \geq 6$. Since $\operatorname{Res}_{X}^{\mathrm{A}_{n}} V$ is irreducible, by [GLT, Theorem 5.1] we have

$$
2^{\frac{n-\lambda_{1}}{2}-1} \leq \operatorname{dim} V \leq \sqrt{\left|\mathrm{A}_{m}\right|}<\sqrt{\frac{m^{m}}{2^{m+1}}}
$$

whence $n-\lambda_{1}<m \log _{2} m-m+1$. This in turn implies that $2 \lambda_{1}-n \geq n / m$. Indeed, otherwise we would have $\lambda_{1}<n / 2+n / 2 m$ and so

$$
n-\lambda_{1}>\frac{n}{2}-\frac{n}{2 m}=n \cdot \frac{m-1}{2 m} \geq \frac{(m-1)^{2}}{2}>m \log _{2} m-m+1,
$$

a contradiction.

We have shown that $2 \lambda_{1}-n \geq n / m \geq n / b=a \geq 3$. Also, $n-\lambda_{1} \geq 1$ since $\operatorname{dim} V>1$. Applying Proposition 4.2, we see that the restriction of $D^{\lambda}$ to a natural subgroup $S_{a}$ of $\mathrm{S}_{n}$ affords both 1 and $D^{(a-1,1)}$ as composition factors. If $a \geq 5$, then $\operatorname{dim} D^{(a-1,1)} \geq 4$ and so any irreducible summand of the $\mathrm{A}_{a}$-module $D^{(a-1,1)}$ has dimension $\geq 2$ (in fact $D^{(a-1,1)}$ is irreducible over $\left.\mathrm{A}_{a}\right)$. On the other hand, if $a=3$, then $D^{(a-1,1)}$ splits into a direct sum of two non-trivial irreducible $\mathrm{A}_{a}$-submodules. Thus $\operatorname{Res}_{\mathrm{A}_{a}} \mathrm{~A}_{n} V$ affords both the trivial module and also another non-trivial irreducible module as composition factors. It follows that $\operatorname{Res}_{Z}^{\mathrm{A}_{n}} V$ cannot be homogeneous for $Z:=\mathrm{A}_{a} \times \ldots \times \mathrm{A}_{a}=\left(\mathrm{A}_{a}\right)^{b} \triangleleft Y$. But this is a contradiction, as $Z \triangleleft Y$ and the $Y$-module $V$ is primitive.

The following lemma deals with tensor indecomposable irreducible representations of $\mathrm{A}_{m}$. These were studied extensively in [Z, BeK1, BeK2, GoK, GrJ, BeK3].

Lemma 8.7. Let $X \cong \mathrm{A}_{m}$ be a subgroup of $\mathrm{A}_{n}$ with $n \geq m \geq 5$. Let $V$ be an irreducible $\mathbb{F} \mathrm{A}_{n}$-module of dimension $>1$. Assume that the $X$-module $\operatorname{Res}_{X}^{\mathrm{A}_{n}} V$ is irreducible and tensor indecomposable. Then one of the following holds:

(i) $X$ is a transitive subgroup of $\mathrm{A}_{n}$. 
(ii) There is some $t \in\{1,2\}$ such that $X$ fixes $t$ points and acts transitively on $n-t$ remaining points of $\Omega$. Furthermore, $V$ is irreducible over a natural subgroup $\mathrm{A}_{n-t}$ of $\mathrm{A}_{n}$.

Proof. Suppose that $X$ acts intransitively on $\Omega$. Each non-trivial orbit of $X$ has at least $m \geq 5$ points. If $X$ has at least two non-trivial orbits on $X$, then we may assume that there is some $5 \leq k \leq n / 2$ such that $X$ acts non-trivially on both $\Omega^{(1)}:=\{1,2, \ldots, k\}$ and $\Omega^{(2)}:=\{k+1, \ldots, n\}$. These actions induce embeddings $\pi_{i}: X \rightarrow \operatorname{Alt}\left(\Omega^{(i)}\right)$, namely, $g \in X$ acts on $\Omega^{(i)}$ as $\pi_{i}(g)$. Now, $X<Y:=\mathrm{A}_{k} \times \mathrm{A}_{n-k}$ and $\operatorname{Res}_{Y}^{\mathrm{A}_{n}} V$ is irreducible. Hence $\left.V\right|_{Y} \cong V_{1} \otimes V_{2}$ is an outer tensor product of irreducible modules $V_{1}$ over $\mathrm{A}_{k}$ and $V_{2}$ over $\mathrm{A}_{m-k}$. Note that $\operatorname{dim} V_{1}>1$, as otherwise the 3 -cycles in $\mathrm{A}_{k}$ would act trivially on $V$. Similarly $\operatorname{dim} V_{2}>1$. It follows that $\operatorname{Res}_{X}^{\mathrm{A}_{n}} V=U_{1} \otimes U_{2}$, where $U_{i} \cong \operatorname{Res}_{\pi_{i}(X)}^{\mathrm{A}_{k_{i}}} V_{i}$ for $i=1,2$ and $\left(k_{1}, k_{2}\right):=(k, n-k)$. In particular, $\operatorname{Res}_{X}^{\mathrm{A}_{n}} V$ is tensor decomposable, contrary to the assumption.

Thus $X$ has only one non-trivial orbit on $\Omega$, say of length $n-t$ for some $t>0$, and $X$ fixes the remaining $t$ points. Furthermore, $t \leq 2$, as otherwise $X$ is centralized by a 3 -cycle and so cannot act irreducibly on $V$.

Finally, in connection with Lemma 8.7, we bound the number of extensions of irreducible representations of $A_{n-1}$ to $A_{n}$. For this type of question, it is convenient to use the following simple observation:

Lemma 8.8. Let $Y$ be a subgroup of $X$ and $U$ be a finite-dimensional vector space over $\mathbb{F}$. Let $\Psi: Y \rightarrow G L(U)$ be an irreducible representation. Suppose that $\Phi_{i}: X \rightarrow G L(U)$, $i=1,2$, are two isomorphic representations of $X$ with $\operatorname{Res}_{Y}^{X}\left(\Phi_{i}\right)=\Psi$. Then in fact $\Phi_{1}=\Phi_{2}$.

Proof. Fix a basis of the vector space $U$ and write $\Psi$ and $\Phi_{i}$ as matrix representations wit respect to this basis. By the assumption, there is some invertible matrix $A$ such that $\Phi_{2}(x)=A \Phi_{1}(x) A^{-1}$ for all $x \in X$. Since both $\Phi_{i}$ extend $\Psi$, we have that $\Psi(y)=$ $A \Psi(y) A^{-1}$ for all $y \in Y$. By Schur's lemma, $A$ is scalar, and so $\Phi_{2}=\Phi_{1}$.

Now we deal with the aforementioned question for symmetric groups:

Lemma 8.9. Assume $n \geq 3$ and fix a natural embedding of $\mathrm{S}_{n-1}$ into $\mathrm{S}_{n}$. Then every irreducible $\mathbb{F} \mathrm{S}_{n-1}$-representation $\mathrm{S}_{n-1} \rightarrow G L(U)$ has at most one extension to $\mathrm{S}_{n}$.

Proof. Consider $U$ as an $\mathbb{F S}_{n-1}$-module, and suppose that there are two distinct $p$-regular partitions $\lambda, \mu \vdash n$ such that

$$
\operatorname{Res}_{\mathrm{S}_{n-1}}^{\mathrm{S}_{n}}\left(D^{\lambda}\right) \cong \operatorname{Res}_{\mathrm{S}_{n-1}}^{\mathrm{S}_{n}}\left(D^{\mu}\right) \cong U .
$$

Since $\operatorname{soc}\left(\operatorname{Res}_{\mathrm{S}_{n-1}}^{\mathrm{S}_{n}}\left(D^{\lambda}\right)\right) \cong \operatorname{soc}\left(\operatorname{Res}_{\mathrm{S}_{n-1}}^{\mathrm{S}_{n}}\left(D^{\mu}\right)\right)$, by [K3, Corollary 5.3], precisely one of the modules $\operatorname{Res}_{S_{n-1}}^{S_{n}}\left(D^{\lambda}\right), \operatorname{Res}_{S_{n-1}}^{S_{n}}\left(D^{\mu}\right)$ is reducible, a contradiction. Now apply Lemma 8.8 .

Proposition 8.10. Assume $n \geq 5$ and fix a natural embedding of $\mathrm{A}_{n-1}$ into $\mathrm{A}_{n}$. Then every irreducible $\mathbb{F} \mathrm{A}_{n-1}$-representation $\mathrm{A}_{n-1} \rightarrow G L(U)$ has at most three distinct extensions to $\mathrm{A}_{n}$. 
Proof. We assume that $U$ extends to an $\mathbb{F} \mathrm{A}_{n}$-module $V_{1}$ and find all possible other extensions $V_{2}$ of $U$ to $\mathrm{A}_{n}$. Consider $\mathrm{A}_{n-1}$ as the derived subgroup of a natural subgroup $\mathrm{S}_{n-1}$ of $\mathrm{S}_{n}$. By Lemma 8.8, it suffices to bound the number of extensions up to isomorphism. For each $i=1,2$, we can find an irreducible $\mathbb{F} S_{n}$-module $W_{i}$ such that $V_{i} \hookrightarrow \operatorname{soc}\left(\operatorname{Res}_{\mathrm{A}_{n}}^{\mathrm{S}_{n}}\left(W_{i}\right)\right)$, and an irreducible $\mathbb{F} S_{n-1}$-module $T$ such that $U \hookrightarrow \operatorname{soc}\left(\operatorname{Res}_{\mathrm{A}_{n-1}}^{\mathrm{S}_{n-1}}(T)\right)$. We also fix a transposition $g \in \mathrm{S}_{n-1} \backslash \mathrm{A}_{n-1}$, and distinguish the following cases.

Case I: $U$ is not $\mathrm{S}_{n-1}$-invariant. Then for $i=1,2$ we have that

$$
\operatorname{Res}_{\mathrm{A}_{n-1}}^{\mathrm{A}_{n}}\left(V_{i}^{g}\right)=U^{g} \neq U=\operatorname{Res}_{\mathrm{A}_{n-1}}^{\mathrm{A}_{n}}\left(V_{i}\right),
$$

and so $V_{i}$ is not $\mathrm{S}_{n}$-invariant. Hence $\operatorname{Res}_{\mathrm{A}_{n}}^{\mathrm{S}_{n}}\left(W_{i}\right) \cong V_{i} \oplus V_{i}^{g}$, and similarly $\operatorname{Res}_{\mathrm{A}_{n-1}}^{\mathrm{S}_{n-1}}(T) \cong$ $U \oplus U^{g}$. Now $\operatorname{Res}_{\mathrm{A}_{n-1}}^{\mathrm{S}_{n}}\left(W_{i}\right) \cong U \oplus U^{g} \cong \operatorname{Res}_{\mathrm{A}_{n-1}}^{\mathrm{S}_{n-1}}(T)$. It follows that $\operatorname{Res}_{\mathrm{S}_{n-1}}^{\mathrm{S}_{n}}\left(W_{1}\right) \cong T \cong$ $\operatorname{Res}_{\mathrm{S}_{n-1}}^{\mathrm{S}_{n}}\left(W_{2}\right)$. By Lemma 8.9, $W_{1} \cong W_{2}$, whence $V_{2}$ is isomorphic to $V_{1}$ or $V_{1}^{g}$. Since $\operatorname{Res}_{\mathrm{A}_{n-1}}^{\mathrm{A}_{n}}\left(V_{1}^{g}\right)=U^{g} ¥ U$, we must have that $V_{2} \cong V_{1}$.

Case IIa: $U$ is $S_{n-1}$-invariant and both $V_{1}, V_{2}$ are $S_{n}$-invariant. In particular, we have $\operatorname{Res}_{\mathrm{A}_{n}}\left(W_{i}\right)=V_{i}$ for $i=1,2$ and similarly $\operatorname{Res}_{\mathrm{A}_{n-1}} \mathrm{~S}_{n-1}(T)=U$. Now if $p=2$, then the Brauer character of any extension of $U$ to $S_{n-1}$ is uniquely determined by its restriction to $\mathrm{A}_{n-1}$ which is the Brauer character of $U$. Thus $U$ has a unique extension to $S_{n-1}$, and so $\operatorname{Res}_{\mathrm{S}_{n-1}}^{\mathrm{S}_{n}}\left(W_{1}\right) \cong \operatorname{Res}_{\mathrm{S}_{n-1}}^{\mathrm{S}_{n}}\left(W_{2}\right)$. It follows by Lemma 8.9 that $W_{1} \cong W_{2}$ and so $V_{1} \cong V_{2}$.

Next suppose that $p \neq 2$. Then $U$ has two distinct extensions $T$ and $T^{g}$ to $\mathrm{S}_{n-1}$ and $V_{i}$ has two distinct extensions $W_{i}$ and $W_{i}^{g}$ to $\mathrm{S}_{n}$. It follows that $\operatorname{Res}_{\mathbf{S}_{n-1}} \mathbf{S}_{n}\left(W_{i}\right) \in\left\{T, T^{g}\right\}$ and so $\operatorname{Res}_{\mathrm{S}_{n-1}}^{\mathrm{S}_{n}}\left(W_{2}\right) \in\left\{\operatorname{Res}_{\mathrm{S}_{n-1}}^{\mathrm{S}_{n}}\left(W_{1}\right), \operatorname{Res}_{\mathrm{S}_{n-1}}^{\mathrm{S}_{n}}\left(W_{1}^{g}\right)\right\}$. By Lemma 8.9, $W_{2} \cong W_{1}$ or $W_{1}^{g}$, and so $V_{2} \cong V_{1}$.

Thus Case IIa shows that among the extensions of $U$ to $\mathrm{A}_{n}$, at most one of them is $\mathrm{S}_{n}$-invariant.

Case IIb: $U$ is $\mathrm{S}_{n-1}$-invariant, but neither $V_{1}$ nor $V_{2}$ is $\mathrm{S}_{n}$-invariant. In this case for $i=1,2$ we have that $W_{i}=\operatorname{Ind}_{\mathrm{A}_{n}}^{\mathrm{S}_{n}}\left(V_{i}\right)$, and so

$$
\operatorname{Res}_{\mathrm{S}_{n-1}}^{\mathrm{S}_{n}}\left(W_{i}\right) \cong \operatorname{Ind}_{\mathrm{A}_{n-1}}^{\mathrm{S}_{n-1}}\left(\operatorname{Res}_{\mathrm{A}_{n-1}}^{\mathrm{A}_{n}}\left(V_{i}\right)\right) \cong \operatorname{Ind}_{\mathrm{A}_{n-1}}^{\mathrm{S}_{n-1}}(U)
$$

is reducible. Also, $\operatorname{soc}\left(\operatorname{Res}_{S_{n-1}}^{S_{n}}\left(W_{1}\right)\right) \cong \operatorname{soc}\left(\operatorname{Res}_{S_{n-1}}^{S_{n}}\left(W_{2}\right)\right)$. If moreover $W_{1} \not W_{2}$, then the latter implies by [K3, Corollary 5.3] that (precisely) one of the modules $\operatorname{Res}_{\mathrm{S}_{n-1}}\left(W_{i}\right)$, $i=1,2$, is irreducible, a contradiction. It follows that $W_{1} \cong W_{2}$ and so $V_{2} \in\left\{V_{1}, V_{1}^{g}\right\}$ since $\operatorname{Res}_{\mathrm{A}_{n}}^{\mathrm{S}_{n}}\left(W_{1}\right)=V_{1} \oplus V_{1}^{g}$.

Thus Case IIb shows that among the extensions of $U$ to $\mathrm{A}_{n}$, at most two of them can be non- $S_{n}$-invariant. Hence in the case where $U$ is $S_{n-1}$-invariant, there are at most three extensions to $\mathrm{A}_{n}$.

\section{RANK 3 PERMUtATION GROUPS}

To illustrate applicability of Theorem 6.5 to other primitive subgroups of $\mathrm{S}_{n}$, in this section we consider finite simple classical groups $X$ acting as rank 3 permutation groups on $\Omega$, where $(\Omega, X)$ is one of the following: 
(i) $\Omega$ is the set of 2-dimensional subspaces of $W=\mathbb{F}_{q}^{d}$ and $X=P S L(W)=P S L_{d}(q)$ with $d \geq 4$;

(ii) $\Omega$ is the set of singular 1-dimensional subspaces of $W=\mathbb{F}_{q}^{d}$ and $X=P S p(W)^{\prime}=$ $P S p_{d}(q)^{\prime}$ with $2 \mid d \geq 4$ (note that $S p_{d}(q)^{\prime} \neq S p_{d}(q)$ only when $(d, q)=(4,2)$ );

(iii) $\Omega$ is the set of singular 1-dimensional subspaces of $W=\mathbb{F}_{q}^{d}$ and $X=P \Omega(W)=$ $P \Omega_{d}^{ \pm}(q)$ with $d \geq 5$

(iv) $\Omega$ is the set of singular 1-dimensional subspaces of $W=\mathbb{F}_{q^{2}}^{d}$ and $X=P S U(W)=$ $P S U_{d}(q)$ with $d \geq 4$.

According to the main result of [KaL], these families account for all the standard rank 3 permutation representations of finite simple classical groups.

Lemma 9.1. Let $X<\operatorname{Sym}(\Omega)=\mathrm{S}_{n}$, where $(\Omega, X)$ is as listed above, and $2 \mid n$.

(i) Assume furthermore that $d \geq 6$ when $X=P S p_{d}(q)$ or $X=P S U_{d}(q)$, and $d \geq 7$ when $X=P \Omega_{d}^{ \pm}(q)$. Then $f_{3}(X) \geq 6$.

(ii) Also, $f_{3}(X) \geq 5$ if $X=P S p_{4}(q), P S U_{4}(q), P S U_{5}(q), \Omega_{5}(q)$, or $P \Omega_{6}^{ \pm}(q)$.

Proof. (a) First we consider the case $X=P S L(W)$ with $W=\left\langle e_{1}, \ldots, e_{d}\right\rangle_{\mathbb{F}_{q}}$ of dimension $d \geq 4$. Then $X$ has at least six orbits on $\Omega_{3}$, with the following representatives:

- $A=\left\langle e_{1}, e_{2}\right\rangle_{\mathbb{F}_{q}}, B=\left\langle e_{1}, e_{3}\right\rangle_{\mathbb{F}_{q}}, C=\left\langle e_{2}, e_{3}\right\rangle_{\mathbb{F}_{q}}$ (note that $\operatorname{dim}_{\mathbb{F}_{q}}(A+B+C)=3$ and $A \cap B \cap C=0$ here);

- $A=\left\langle e_{1}, e_{2}\right\rangle_{\mathbb{F}_{q}}, B=\left\langle e_{1}, e_{3}\right\rangle_{\mathbb{F}_{q}}, C=\left\langle e_{1}, e_{2}+e_{3}\right\rangle_{\mathbb{F}_{q}}$ (here, $\operatorname{dim}_{\mathbb{F}_{q}}(A+B+C)=3$ and $A \cap B \cap C \neq 0)$;

- $A=\left\langle e_{1}, e_{2}\right\rangle_{\mathbb{F}_{q}}, B=\left\langle e_{3}, e_{4}\right\rangle_{\mathbb{F}_{q}}, C=\left\langle e_{1}+e_{3}, e_{2}+e_{4}\right\rangle_{\mathbb{F}_{q}}$ (here, $\operatorname{dim}_{\mathbb{F}_{q}}(A+B+C)=4$ and $\left\{\operatorname{dim}_{\mathbb{F}_{q}}(A \cap B), \operatorname{dim}_{\mathbb{F}_{q}}(A \cap C), \operatorname{dim}_{\mathbb{F}_{q}}(B \cap C)=\{0,0,0\}\right)$;

- $A=\left\langle e_{1}, e_{2}\right\rangle_{\mathbb{F}_{q}}, B=\left\langle e_{1}, e_{3}\right\rangle_{\mathbb{F}_{q}}, C=\left\langle e_{2}+e_{3}, e_{4}\right\rangle_{\mathbb{F}_{q}}$ (here, $\operatorname{dim}_{\mathbb{F}_{q}}(A+B+C)=4$ and $\left\{\operatorname{dim}_{\mathbb{F}_{q}}(A \cap B), \operatorname{dim}_{\mathbb{F}_{q}}(A \cap C), \operatorname{dim}_{\mathbb{F}_{q}}(B \cap C)=\{1,0,0\}\right) ;$

- $A=\left\langle e_{1}, e_{2}\right\rangle_{\mathbb{F}_{q}}, B=\left\langle e_{1}, e_{3}\right\rangle_{\mathbb{F}_{q}}, C=\left\langle e_{2}, e_{4}\right\rangle_{\mathbb{F}_{q}}$ (here, $\operatorname{dim}_{\mathbb{F}_{q}}(A+B+C)=4$ and $\left\{\operatorname{dim}_{\mathbb{F}_{q}}(A \cap B), \operatorname{dim}_{\mathbb{F}_{q}}(A \cap C), \operatorname{dim}_{\mathbb{F}_{q}}(B \cap C)=\{1,1,0\}\right) ;$

- $A=\left\langle e_{1}, e_{2}\right\rangle_{\mathbb{F}_{q}}, B=\left\langle e_{1}, e_{3}\right\rangle_{\mathbb{F}_{q}}, C=\left\langle e_{1}, e_{4}\right\rangle_{\mathbb{F}_{q}}$ (here, $\operatorname{dim}_{\mathbb{F}_{q}}(A+B+C)=4$ and $\left.\operatorname{dim}_{\mathbb{F}_{q}}(A \cap B \cap C)=1\right)$.

(b) In the remaining cases of (i), the assumption on $d$ implies that $W$ contains a non-degenerate 6 -dimensional subspace with hyperbolic (Witt) basis $\left(e_{1}, e_{2}, e_{3}, f_{1}, f_{2}, f_{3}\right)$. Also, since $n=|\Omega|$ is even, $q$ is odd. We will write any element in $\Omega$ as $\langle a\rangle$, the 1 -space generated by a vector $a \in W$. In the $\Omega$-case, for any unordered triple $\pi=\{A, B, C\}$, with $A=\langle a\rangle, B=\langle b\rangle, C=\langle c\rangle \in \Omega$ and $\operatorname{dim}(A+B+C)=3$, we can associate to it the Gram matrix $\Gamma$ of the bilinear form $(\cdot, \cdot)$ written in the basis $(a, b, c)$. Since changing to another basis of $A+B+C$ changes $\operatorname{det}(\Gamma)$ by a factor which belongs to the subgroup $F_{0}:=\mathbb{F}_{q}^{\times 2}$ of $F:=\mathbb{F}_{q}^{\times}$, we can associate to such $\pi$ a canonical element $\delta:=\operatorname{det}(\Gamma) F_{0} \in F / F_{0}$. Then the following unordered triples $\{A, B, C\} \in \Omega_{3}$ belong to disjoint $X$-orbits:

- $A=\left\langle e_{1}\right\rangle, B=\left\langle e_{2}\right\rangle, C=\left\langle e_{1}+e_{2}\right\rangle$ (note that $A+B+C$ is a 2-dimensional totally singular subspace here);

- In the $S p / S U$-case: $A=\left\langle e_{1}\right\rangle, B=\left\langle f_{1}\right\rangle, C=\left\langle e_{1}+\lambda f_{1}\right\rangle$, where $\lambda=1$ in the $S p$-case and $\lambda^{q-1}=-1$ in the $S U$-case. Note that $A+B+C$ is a 2-dimensional non-degenerate subspace here; 
- $A=\left\langle e_{1}\right\rangle, B=\left\langle e_{2}\right\rangle, C=\left\langle e_{3}\right\rangle$ (here, $A+B+C$ is a 3-dimensional totally singular subspace);

- $A=\left\langle e_{1}\right\rangle, B=\left\langle f_{1}\right\rangle, C=\left\langle e_{2}\right\rangle$. Note that $U:=A+B+C$ is a 3 -dimensional subspace with $C=\operatorname{rad}(U)$;

- $A=\left\langle e_{1}\right\rangle, B=\left\langle f_{1}\right\rangle, C=\left\langle e_{1}+e_{2}\right\rangle$. Note that $U:=A+B+C$ is a 3-dimensional subspace with $\operatorname{dim} \operatorname{rad}(U)=1$ but $A, B, C \neq \operatorname{rad}(U)$. In fact, in the $S p$-case, we get one more triple with the same $A, B$, but with $C^{\prime}=\left\langle e_{1}+f_{1}+e_{2}\right\rangle$ - note that $A \perp C$, but no two of $A, B, C^{\prime}$ are orthogonal to each other;

- In the $\Omega / S U$-case: $A=\left\langle e_{1}\right\rangle, B=\left\langle f_{1}\right\rangle, C=\left\langle e_{2}+\lambda f_{2}+e_{1}-\lambda f_{1}\right\rangle$, where $\lambda \in F$ in the $\Omega$-case and $\lambda=1$ in the $S U$-case. Here, $W:=A+B+C$ is a 3 -dimensional non-degenerate subspace. Furthermore, in the $\Omega$-case, we have $\delta=-2 \lambda F_{0}$, whence we can choose $\lambda$ so that $\delta=F_{0}$, respectively $\delta \neq F_{0}$.

(c) Ignoring the vectors $e_{3}, f_{3}$, the arguments in (b) also show that $f_{2}(X) \geq 5$ if $X=P S p_{4}(q), P S U_{4}(q), P S U_{5}(q), \Omega_{5}(q)$, or $\Omega_{6}^{ \pm}(q)$.

Lemma 9.2. Let $X<\operatorname{Sym}(\Omega)=\mathrm{S}_{n}$, where $(\Omega, X)$ is as listed above, that is, $d \geq 4$ when $X=P S L_{d}(q), P S U_{d}(q)$, or $P S p_{d}(q)$, and $d \geq 5$ when $X=P \Omega_{d}^{ \pm}(q)$. Suppose that $p=2 \mid n$. Then $h(X) \leq 3$ if $X=P \Omega_{d}^{+}(q)$ with $4 \mid d \geq 8$, and $h(X) \leq 2$ otherwise.

Proof. As mentioned in the proof of Lemma 9.1, $2 \mid n$ implies that $q$ is odd. We follow the proof of Lemma 7.2 and its notation. First, $\operatorname{dim} H^{2}(X, \mathbb{F})$, which is the 2-rank of the Schur multiplier of $X$, is $\leq 2$ if $X=P \Omega_{d}^{+}(q)$ with $4 \mid d \geq 8$, and $\leq 1$ otherwise by [KlL, Theorem 5.1.4]. Hence it suffices to show that

$$
\operatorname{dim} \operatorname{Hom}\left(X_{1},(\mathbb{F},+)\right) \leq 1,
$$

where $X_{1}$ is the point stabilizer in $X$ of a point in $\Omega$. Without loss we may replace $X$ by its central cover $S L_{d}(q), S U_{d}(q), S p_{d}(q)$, or $\Omega_{d}^{ \pm}(q)$. We also fix a basis $\left(e_{1}, \ldots, e_{d}\right)$ of the natural module $W$ of $X$.

Consider the case $X=S L_{d}(q)$. Then $X_{1}=\operatorname{Stab}_{X}\left(\left\langle e_{1}, e_{2}\right\rangle\right)=Q \rtimes Y$, where $|Q|$ is a $q$-power and $Y=\left(S L_{2}(q) \times S L_{d-2}(q)\right) \rtimes C_{q-1}$. Since $q$ is odd, we have that $O^{2}(Q)=Q$ and $O^{2}\left(S L_{e}(q)\right)=S L_{e}(q)$ for any $e \geq 2$. Hence (9.1) follows.

From now on we may assume $X \neq S L_{d}(q)$. We can then choose $e_{1}$ to be singular and let $X_{1}=\operatorname{Stab}_{X}\left(\left\langle e_{1}\right\rangle\right)$.

Let $X=S U_{d}(q)$. Then $X_{1}=Q \rtimes Y$, where $|Q|$ is a $q$-power and $Y=S U_{d-2}(q) \rtimes C_{q^{2}-1}$. As $d \geq 4$ and $q$ is odd, we see that $O^{2}\left(S U_{d-2}(q)\right)=S U_{d-2}(q)$, and so (9.1) follows.

Suppose $X=S p_{d}(q)$. Then $X_{1}=Q \rtimes Y$, where $|Q|$ is a $q$-power and $Y=S p_{d-2}(q) \rtimes$ $C_{q-1}$. As $d \geq 4$ and $q$ is odd, we see that $O^{2}\left(S p_{d-2}(q)\right)=S p_{d-2}(q)$, yielding (9.1).

Suppose $X=\Omega_{d}^{\epsilon}(q)$. Then $X_{1}=Q \rtimes Y$, where $|Q|$ is a $q$-power and $Y=\Omega_{d-2}^{\epsilon}(q) \rtimes$ $C_{q-1}$. As $d \geq 5$ and $q$ is odd, we see that $O^{2}\left(\Omega_{d-2}^{\epsilon}(q)\right)=\Omega_{d-2}^{\epsilon}(q)$, and so we are done.

Proof of Theorem 1.3. Assume the contrary: $\operatorname{Res}_{X}^{A_{n}} V$ is irreducible; in particular, $V$ is irreducible. Note that $X$ is not 2-transitive on $\Omega$. Hence, by the main results of [KS1, KS2] we must have that $p=2$ or 3 .

(i) In the case $X=P \operatorname{Sp}_{4}(2)^{\prime} \cong \mathrm{A}_{6}$, we have $n=15$, and so $\operatorname{dim} V \geq 13$ (see [Dec]), whereas the largest dimension $\mathfrak{b}(X)$ of irreducible $\mathbb{F} X$-modules is at most 10, cf. [JLPW]. 
Thus $V$ is reducible over $X$. Next, in the cases $X=S U_{4}(2) \cong P S p_{4}(3)$, respectively $S L_{4}(2), S p_{6}(2)$, we have $n \geq 27,35,63$, and $\mathfrak{b}(X) \leq 81,70,512$, respectively, according to [JLPW]. Certainly, $\operatorname{Res}_{X}^{\bar{A}_{n}} V$ is reducible if $\operatorname{dim} V>\mathfrak{b}(X)$. So we must have that $\operatorname{dim} V \leq \mathfrak{b}(X)$. Since $\mathfrak{b}(X)<\left(n^{2}-5 n+2\right) / 2$, by [GT1, Lemma 6.1] we see that $V \cong D^{(n-1,1)}$ is isomorphic to the heart of the natural permutation module $\mathbb{F} \Omega$. As in the proof of Lemma 8.1, we conclude that $X$ is 2 -transitive on $\Omega$, a contradiction.

(ii) We may now assume that $X$ is not isomorphic to any of the groups considered in (i). Direct computation shows that $2^{(n-8) / 4}>|X|^{1 / 2}$. Since $\operatorname{Res}_{X}^{\mathrm{A}_{n}} V$ is irreducible, we must have that

$$
\operatorname{dim}(V)<2^{(n-8) / 4}
$$

which implies by Propositions 4.1 and 4.3 that $V$ extends to $\mathrm{S}_{n}$. Applying the Main Theorem and Theorem 3.10 of [KS1], we again arrive at the contradiction that $X$ is 2-transitive in the case $p=3$, as well as in the case $p=2 \nmid n$.

Thus we have shown that $p=2 \mid n$. The upper bound (9.2) implies by Theorem 3.2 that $d_{3}(V)>d_{1}(V)$. Also, $f_{1}(X)=1, f_{2}(X)=2$ by Lemma 6.3 , and $e_{3}(X) \geq h(X)+1$ by Lemmas 9.1 and 9.2. Now we can apply Theorem 6.5.

Non-standard rank 3 permutation representations of finite classical groups, as well as other primitive subgroups of $A_{n}$, will be considered elsewhere.

\section{REFERENCES}

[A] M. Aschbacher, On the maximal subgroups of the finite classical groups, Invent. Math. 76 (1984), 469-514.

[AS] M. Aschbacher and L. Scott, Maximal subgroups of finite groups, J. Algebra 92 (1985), 44-80.

$[\mathrm{BaK}] \quad$ A. Baranov and A.S. Kleshchev, Maximal ideals in modular group algebras of the finitary symmetric and alternating groups, Trans. Amer. Math. Soc. 351 (1999), 595-617.

[Ben] D. Benson, Spin modules for symmetric groups, J. London Math. Soc. 38 (1988), 250-262.

[BeK1] C. Bessenrodt and A.S. Kleshchev, On Kronecker products of complex representations of the symmetric and alternating groups, Pacific J. Math. 190 (1999), 201-223.

[BeK2] C. Bessenrodt and A.S. Kleshchev, On tensor products of modular representations of symmetric groups, Bull. London Math. Soc. 32 (2000), 292-296.

[BeK3] C. Bessenrodt and A.S. Kleshchev, Irreducible tensor products over alternating groups, J. Algebra 228 (2000), 536-550.

[BeO] C. Bessenrodt and J.B. Olsson, On residue symbols and the Mullineux conjecture, J. Alg. Comb. 7 (1998), 227-251.

[BDR] J.N. Bray, D.F. Holt, and C.M. Roney-Dougal, 'The Maximal Subgroups of the Low-dimensional Finite Classical Groups', London Math. Soc. Lecture Note Series no. 407. Camb. Univ. Press, 2013.

[BK] J. Brundan and A.S. Kleshchev, Representations of the symmetric group which are irreducible over subgroups, J. Reine Angew. Math. 530 (2001), 145-190.

[Atl] J.H. Conway, R.T. Curtis, S.P. Norton, R.A. Parker, and R.A. Wilson, 'An ATLAS of Finite Groups', Clarendon Press, Oxford, 1985.

[Dec] Decomposition matrices, http://www.math.rwth-aachen.de/homes/MOC/decomposition/

[FK] B. Ford and A.S. Kleshchev, A proof of the Mullineux conjecture, Math. Z. 226 (1997), 267-308.

[GAP] The GAP group, 'GAP - Groups, Algorithms, and Programming', Version 4.4, 2004, http://www .gap-system. org. 
[GoK] R. Gow and A. Kleshchev, Connections between the representations of the symmetric group and the symplectic group in characteristic 2, J. Algebra 221 (1999), 60-89.

[GrJ] J. Graham and G. James, On a conjecture of Gow and Kleshchev concerning tensor products, J. Algebra 227 (2000), 767-782.

[GLT] R.M. Guralnick, M. Larsen, and Pham Huu Tiep, Representation growth in positive characteristic and conjugacy classes of maximal subgroups, Duke Math. J. 161 (2012), 107-137.

[GT1] R.M. Guralnick and Pham Huu Tiep, The non-coprime $k(G V)$ problem, J. Algebra 293 (2005), 185-242.

[GT2] R.M. Guralnick and Pham Huu Tiep, A problem of Kollár and Larsen on finite linear groups and crepant resolutions, J. Europ. Math. Soc. 14 (2012), 605-657.

[J1] G.D. James, Representations of the symmetric group over the field of order 2, J. Algebra 38 (1976), 280-308.

[J2] G.D. James, 'The Representation Theory of the Symmetric Groups', Lecture Notes in Mathematics, vol. 682, Springer, NewYork/Heidelberg/Berlin, 1978.

[J3] G.D. James, On the minimal dimensions of irreducible representations of symmetric groups, Math. Proc. Camb. Phil. Soc. 94 (1983), 417-424.

[JLPW] C. Jansen, K. Lux, R.A. Parker, and R.A. Wilson, 'An ATLAS of Brauer Characters', Oxford University Press, Oxford, 1995.

[KaL] W.M. Kantor and R. Liebler, The rank 3 permutation representations of the finite classical groups, Trans. Amer. Math. Soc. 271 (1982), 1-71.

[KlL] P.B. Kleidman and M.W. Liebeck, 'The Subgroup Structure of the Finite Classical Groups', London Math. Soc. Lecture Note Ser. no. 129, Camb. Univ. Press, 1990.

[KlW] P.B. Kleidman and D.B. Wales, The projective characters of the symmetric groups that remain irreducible on subgroups, J. Algebra 138 (1991), 440-478.

[K1] A.S. Kleshchev, On restrictions of irreducible modular representations of semisimple algebraic groups and symmetric groups to some natural subgroups. I, Proc. Lond. Math. Soc. (3) 69 (1994), 515-540.

[K2] A.S. Kleshchev, Branching rules for modular representations of symmetric groups. II, J. Reine Angew. Math. 459 (1995), 163-212.

[K3] A.S. Kleshchev, Branching rules for modular representations of symmetric groups. III. Some corollaries and a problem of Mullineux, J. London Math. Soc. 54 (1996), 25-38.

[KS1] A.S. Kleshchev and J. Sheth, Representations of the symmetric group are reducible over simply transitive subgroups, Math. Z. 235 (2000), 99-109.

[KS2] A.S. Kleshchev and J. Sheth, Representations of the alternating group which are irreducible over subgroups, Proc. London Math. Soc. 84 (2002), 194-212.

[KT1] A.S. Kleshchev and Pham Huu Tiep, On restrictions of modular spin representations of symmetric and alternating groups, Trans. Amer. Math. Soc. 356 (2004), 1971-1999.

[KT2] A.S. Kleshchev and Pham Huu Tiep, Representations of the general linear groups which are irreducible over subgroups, Amer. J. Math. 132 (2010), 425-473.

[LPS] M.W. Liebeck, C. Praeger, and J. Saxl, A classification of the maximal subgroups of the finite alternating groups and symmetric groups, J. Algebra 111 (1987), 365-383.

[LS] M.W. Liebeck and G. M. Seitz, On finite subgroups of exceptional algebraic groups, J. reine angew. Math. 515 (1999), 25-72.

[Ma] E. Maillet, Sur les isomorphes holoédriques et transitifs des groupes symétriques ou alternés, $J$. Math. Pures Appl. 1 (1895), 5-34.

[Mo] B. Mortimer, The modular permutation representations of the known doubly transitive groups, Proc. London Math. Soc. (3) 41(1980), 1-20.

[MO] J. Müller and J. Orlob, On the structure of the tensor square of the natural module of the symmetric group, Algebra Colloq. 18 (2011), 589-610.

$[\mathrm{Mu}] \quad$ G. Mullineux, Bijections of $p$-regular partitions and $p$-modular irreducibles of the symmetric groups, J. London Math. Soc. 20 (1979), 60-66. 
[P] A.M. Phillips, On 2-modular representations of the symmetric groups, J. Algebra, 290 (2005), $282-294$.

[S] J. Saxl, The complex characters of the symmetric groups that remain irreducible in subgroups, J. Algebra 111 (1987), 210-219.

[W] D.B. Wales, Some projective representations of $S_{n}$, J. Algebra 61 (1979), 37-57.

[Wie] H. Wielandt, 'Finite Permutation Groups', Academic Press, 1964, 114 pp.

[Wil] R.M. Wilson, A diagonal form for the incidence matrices of $t$-subsets vs. $k$-subsets, European $J$. Combinatorics 11 (1990), 609-615.

[Z] I. Zisser, Irreducible products of characters in $A_{n}$, Israel J. Math. 84 (1993), 147-151.

Department of Mathematics, University of Oregon, Eugene, OR 97403, USA

E-mail address: klesh@uoregon.edu

Department of Mathematics, University of Florida, Gainesville FL 32611-8105, USA

E-mail address: sin@ufl.edu

Department of Mathematics, University of Arizona, Tucson, AZ 85721, USA

E-mail address: tiep@math.arizona.edu 